\title{
Mechanical conditions for stable symmetric cell constriction
}

\author{
Elena Beltrán-Heredia $\odot,{ }^{1,2}$ Francisco Monroy $\odot,{ }^{2,3}$ and Francisco J. Cao-García ${ }^{1,4, *}$ \\ ${ }^{1}$ Departamento de Estructura de la Materia, Física Térmica, y Electrónica, Universidad Complutense de Madrid, Plaza de Ciencias 1, \\ 28040 Madrid, Spain \\ ${ }^{2}$ Departamento de Química Física, Universidad Complutense de Madrid, Avenida Complutense s/n, \\ 28040 Madrid, Spain \\ ${ }^{3}$ Unit of Translational Biophysics, Instituto de Investigación Sanitaria Hospital Doce de Octubre (imas12), Avenida de Cordoba s/n, 28041 \\ Madrid, Spain \\ ${ }^{4}$ IMDEA Nanociencia, Calle Faraday 9, 28049 Madrid, Spain
}

(Received 1 August 2019; revised manuscript received 18 October 2019; published 21 November 2019)

\begin{abstract}
Cell constriction is a decisive step for division in many cells. However, its physical pathway remains poorly understood, calling for a quantitative analysis of the forces required in different cytokinetic scenarios. Using a model cell composed by a flexible membrane (actin cortex and cell membrane) that encloses the cytoplasm, we study the mechanical conditions necessary for stable symmetric constriction under radial equatorial forces using analytical and numerical methods. We deduce that stable symmetric constriction requires positive effective spontaneous curvature, while spontaneous constriction requires a spontaneous curvature higher than the characteristic inverse cell size. Surface tension reduction (for example by actin cortex growth and membrane trafficking) increases the stability and spontaneity of cellular constriction. A reduction of external pressure also increases stability and spontaneity. Cells with prolate lobes (elongated cells) require lower stabilization forces than oblate-shaped cells (discocytes). We also show that the stability and spontaneity of symmetric constriction increase as constriction progresses. Our quantitative results settle the physical requirements for stable cytokinesis, defining a quantitative framework to analyze the mechanical role of the different constriction machinery and cytokinetic pathways found in real cells, so contributing to a deeper quantitative understanding of the physical mechanism of the cell division process.
\end{abstract}

DOI: 10.1103/PhysRevE.100.052408

\section{INTRODUCTION}

Cell division under symmetric constriction is an essential feature of life from which stems the capacity of any living organism for self-replication [1]. No matter what the cell is, either prokaryote or eukaryote, every cell is replicated from preexisting cells through cytokinesis, the terminal process of the cell cycle, and thereby the mother cell is cleaved into two daughters [2]. The key cytokinetic features are conserved in the different kingdoms of life, standing out by their reproducibility and regularity in every organism [2-4]. Such a set of universal characteristics suggests a general mechanical pathway with a tight physical control mediated by specific biomolecular players [5-7]. Specific mechanistic details differ between organisms due to biochemical evolution [8], which makes possible confronting different constriction forces with mutable cells of dissimilar sizes and variable mechanical characteristics [4]. In animal cytokinesis, cell constriction has evolved to occur at an equatorial site specifically named a cleavage furrow [9] [see Fig. 1(a), upper panel]. In plant cells, some structural aspects deviate from the animal cytokinetic scenario; specifically, due to the rigidity of cell walls necessary to support physiological turgor, they form a dividing septum on the equatorial plane of cell division. Cell

*Corresponding author: francao@ucm.es septation, which is the synthesis of the cell wall that separates the daughter cells, is also exploited by prokaryotes [10-16]. Here, we focus on the case of animal eukaryotic cells, as prokaryotes and plant cells require the specific consideration of the mechanical effects of the cell wall where they are encapsulated.

Recent studies and reviews call for the need of an integrated mechanical model of animal cell division $[17,18]$. However, the mechanical properties of the cortex present a huge degree of variability between cell types and may change during the cell cycle [18]. Additionally, some of the forcegenerating mechanisms can be redundant or only activated when they are required to control a perturbation-induced instability as discussed in Ref. [17]. Therefore, determining the required forces for stable symmetric constriction, independently of the force-generating mechanism, is expected to be enlightening for a deep understanding of cytokinesis.

We contribute to this aim using a minimal mechanical model, independent of particular mechanisms, which quantifies the relations between the different mechanical contributions required for stable symmetric cell division. This minimal mechanical model has been already used for the determination of constriction forces (both numerically and through a combined variational and perturbative approach) [19-21]. In Sec. II the minimal model is summarized and extended for our present study to compute the stabilization force required for symmetric constriction for nonzero spontaneous curvature, 
(a)

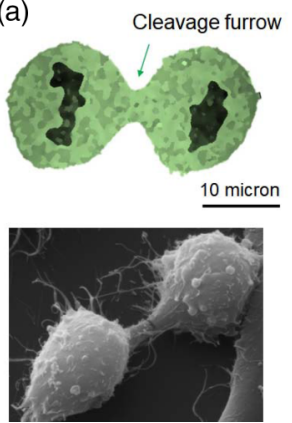

(b)

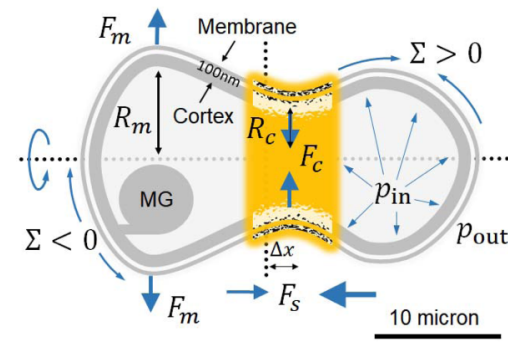

FIG. 1. Real cells and minimal model cell during cytokinesis. (a) HeLa cell undergoing cell constriction (cytokinesis). Upper panel: Formation of the cleavage furrow (optical microscopy image, courtesy of A. Siegel and H. C. Smith, University of Rochester). Lower panel: Scanning electron micrograph during the final stage of constriction (courtesy of A. Wilde, University of Toronto). (b) Profile of a constricted cell and its characteristic parameters. The force $F_{m}$ at the polar radius $R_{m}$ keeps the size of the lobes; the constriction force $F_{c}$ directed toward the cell interior causes constriction to constriction radius $R_{c}$; and the stabilization force $F_{s}$ directed to the middle of the cell $(\Delta x=0)$ symmetrizes the division. External stress fields applied either transversally, as a pressure $\Delta p$, or longitudinally, as a lateral membrane tension $\Sigma$, complete the main systemic factors defining the shape of the cell, whose intrinsic mechanical properties are represented by an effective bending modulus $\kappa$ and an effective spontaneous curvature $C_{0}$.

pressure, and tension. This model entails the mechanical energetics of an effective flexible shell, comprising the plasma membrane and the actin cortex. This cortical layer, whose mechanics is dominated by the actin cortex, is the dominant mechanical ingredient in animal cells [22].

Here, we compute the stabilization forces required for symmetric constriction, independently of their generation mechanisms. These forces are known to be effectively exerted through different mechanisms involving the actin cortex, which include bleb formation to control polar pressure [17], and also spindle position controlling through the microtubulebased motor dynein and asymmetric plasma membrane elongation [23]. Section III presents the results for the stabilization force and compares them with previous results on the constriction force. These results allow us to clarify and compare the conditions for spontaneous constriction and for stable symmetric constriction, and the influence of effective surface tension, pressure, and spontaneous curvature.

\section{THE MODEL CELL}

The natural eukaryotic cell is modeled as a deformable vesicle with the cellular contents enclosed by a flexible shell, which represents the elasticity properties of the cellular membrane and cortex. The total energy of the flexible shell is given in terms of its mean curvature $H$ by the Canham-Helfrich $(\mathrm{CH})$ form [24,25], as

$$
E_{T}=\frac{\kappa}{2} \int_{\Omega}\left(2 H-C_{0}\right)^{2} d A+\Sigma A+\Delta p V .
$$

This CH-model membrane is characterized by an effective spontaneous curvature $C_{0}$ and an effective bending rigidity $\kappa$ to give an effective theory of cell mechanics [26,27]. The spontaneous curvature $C_{0}$ represents the tendency of the membrane to bend in the equilibrium state [28], usually due to the compositional asymmetry between the inner and the outer sides [29]. In our minimal model, $C_{0}$ is assumed to be homogeneous along the whole membrane, thus determining the global shape of the vesicle [30]. This model membrane, of actual surface area $A$, is assumed laterally undeformable and subjected to effective cortical tension $\Sigma$. The cell interior, including cytoplasm, organelles, and other cellular structures, is described as a hydrostatic fluid enclosed in a vesicle of volume $V$ (and surface area $A$ ) subjected to a pressure difference $\Delta p$ between the outer medium and the cell interior $\left(\Delta p=p_{\text {out }}-p_{\text {in }}\right)$. This pressure difference accounts for either possible osmotic imbalances, a turgor pressure, or simply a Laplace pressure due to local curvature [17]. [For additional details see comments on Eq. (A1) in the Appendix.]

Additionally, forces exerted by the cell contents in the cell membrane are simplified to three types of forces: one keeping a certain size of each of the lobes of the cell $F_{m}$, another force exerting constriction in the middle of the cell $F_{c}$, and finally, an effective force stabilizing the symmetric constriction $F_{s}$ [see Fig. 1(b)]. Bulge $F_{m}$ and constriction $F_{c}$ forces are assumed to act as line tensions. Stabilization force $F_{s}$ acts like an effective force centering the constriction region in the middle of the cell. All these forces are effective forces, and each one can have different cellular processes as sources. The effective force acting on the bulge, $F_{m}$, can be due to nucleoid exclusion and the cytoskeleton. The constriction force, $F_{c}$, can be caused by polymerization and depolymerization of actin filaments and to actin cortex flows. Stabilization force, $F_{s}$, includes effective forces exerted by asymmetric cortex flows. Other effects can be sources of these effective forces. However, our approach here is to compute the minimal requirements independently of the character of sources of the forces. Within this approach we consider constriction at constant cell volume (usual traffic membrane case [31-34]) or at constant cell area (inhibited membrane traffic case [19-21,35-38]).

We explore the stability of the symmetric constriction, which will allow us to compute the required stabilization forces. These results are compared with previous results of the required constriction forces. All these results are derived finding the cell shape that minimizes the total energy, Eq. (1), using a combination of perturbative and variational approaches, and verified by numerical computations (for details see below and Refs. [19-21]).

\section{A. Parametrization of the constricted cell profile}

The axisymmetric profile of a symmetrically constricted cell is divided into two different regions: polar caps [left polar cap shaded in blue in Fig. 2(a)] and the constriction zone [left half of the constriction zone shaded in yellow in Fig. 2(a)]. Establishing the origin of the $x$ coordinate in the middle point of the vesicle, the constriction profile can be expressed as

$$
R(x ; s)= \begin{cases}R_{\text {left polar cap }}(x), & \text { if } x \in\left[-L_{p}-L_{m},-L_{m}\right], \\ R_{c z}(x ; s), & \text { if } x \in\left[-L_{m}, L_{m}\right], \\ R_{\text {right polar cap }}(x), & \text { if } x \in\left[L_{m}, L_{m}+L_{p}\right],\end{cases}
$$




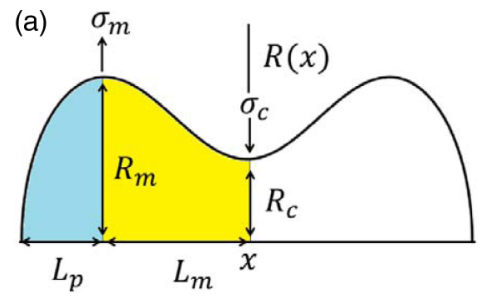

(b)

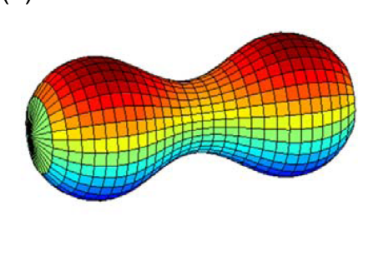

FIG. 2. Symmetrically constricted vesicle. (a) Profile $R(x)$ of a symmetrically constricted vesicle with the axis of symmetry along the $x$ axis and its characteristic parameters. Left polar cap is shaded in blue and the left half of the constriction zone is shaded in yellow. (b) Surface obtained from the revolution around the $x$ axis.

where $L_{m}$ and $L_{p}$ represent half of the length of the constriction zone and the polar distance, respectively [see Fig. 2(a)]. Note that the constriction profile must be continuous in the boundaries of the zones. In addition, since the shape has central symmetry, we have the relation $R_{\text {left pole cap }}(x)=$ $R_{\text {right pole cap }}(-x)$.

The profile describing the right polar cap with semiaxis $R_{m}$ (polar radius) and $L_{p}$ (polar distance) centered at $x=L_{m}$ [see Fig. 2(a)] is

$$
\begin{aligned}
R_{\text {right polar cap }}(x) & = \pm R_{m} \sqrt{1-\left(\frac{x-L_{m}}{L_{p}}\right)^{2}}, \\
x & \in\left[L_{m}, L_{m}+L_{p}\right] .
\end{aligned}
$$

In previous works [5-7], we have seen that the constriction zone can be approximately described with a trigonometric function whose local curvature changes along the constriction process as

$$
R_{c z}(x ; s)=R_{m}\left\{1-\frac{s}{2}\left[1+\cos \left(\frac{\pi x}{L_{m}}\right)\right]\right\},
$$

where the constriction parameter $s$ is defined in terms of the ratio between the constriction radius $R_{c}$ and the polar radius $R_{m}$ in the form $s=1-R_{c} / R_{m}$. The constriction profile of the vesicle $R(x ; s)$ [Eq. (2)], together with its first and second derivatives $\left(R_{x}=\partial R / \partial x\right.$ and $\left.R_{x x}=\partial R_{x} / \partial x\right)$, allows us to determine the minimum energy shapes along the constriction pathway by minimizing the total energy [Eq. (A13) of the Appendix] with respect to the characteristic length of each zone ( $L_{p}$ for the polar caps and $L_{m}$ for the constriction zone):

$$
\begin{gathered}
\frac{\partial E_{T, \text { polar caps }}\left(L_{p}, R_{m}, C_{0}, \Sigma, \Delta p, \kappa\right)}{\partial L_{p}}=0 \stackrel{\text { yields }}{\rightarrow} L_{p}=L_{p}^{\text {opt }}, \quad \text { (5a) } \\
\left.\frac{\partial E_{T, c z}\left(s, L_{m}, R_{m}, C_{0}, \Sigma, \Delta p, \kappa\right)}{\partial L_{m}}\right|_{s}=0 \stackrel{\text { yields }}{\rightarrow} L_{m}(s)=L_{m}^{\text {opt }}(s) .
\end{gathered}
$$

Once optimal total length $L_{p}^{\mathrm{opt}}+L_{m}^{\mathrm{opt}}(s)$ has been obtained, other quantities of the system can be calculated, such as the total energy, the membrane area, the volume enclosed, and the constriction forces. These expressions are given in terms of the spontaneous curvature $C_{0}$, the surface tension $\Sigma$, the osmotic pressure $\Delta p$, the polar radius $R_{m}$, and the constriction parameter $s$ (analytical formulas can be found in Ref. [21]).
The fourth-order expressions describe with $95 \%$ agreement the numerical solution up to constrictions as large as $s \approx 0.65$ (see Figs. 5 and 6 in Ref. [21]). Higher constriction stages require a constriction profile more accurate than Eq. (4) to describe the strong changes of curvature.

When the constriction process is assumed to proceed by keeping the polar radius $R_{m}$ constant, the polar distance $L_{p}$ [Eq. (5a)] becomes independent of the constriction parameter $s$ (and therefore, other properties of the system calculated on the polar caps zone are also independent of $s$ ). Conversely, under constant area or constant volume conditions (see below), these quantities are not constant but vary along the constriction pathway.

In previous works [19-21], the initial cell shape (before constriction) was shown to be determined by spontaneous curvature, surface tension, and external pressure through the quantity

$$
\Lambda=\left(1-C_{0} R_{m}\right)^{2}+2 \Sigma R_{m}^{2} / \kappa+\Delta p R_{m}^{3} / \kappa .
$$

$\Lambda=1$ stands for the sphere, while $0<\Lambda<1$ gives prolate (rodlike) and $\Lambda>1$ oblate (disklike) spheroids; for $\Lambda<$ 0 there are no solutions [21]. The parameter $\Lambda$ also determines the shape of the cell lobes during division (see Fig. 3). Particularly, during constriction prolate-shaped cells (cylinders) elongate along their axis, and constriction becomes favored by lowering the external pressure, or the surface tension (e.g., by enhancing membrane traffic toward the cell membrane). In contrast, oblate cells (discocytes) have flatter shapes, which are favored by high values of external pressure or surface tension (see Fig. 4) (for details see Refs. [19-21]). In general, oblate cells require much stronger constriction forces (see Fig. 4), giving rise to less stable configurations.

The degree of constriction is indicated by the constriction parameter $s=1-R_{c} / R_{m}$, which is defined in terms of the constriction radius $R_{c}$ and the initial cell size measured as a polar radius $R_{m}$ (see Fig. 2). This parameter increases from $s=0$, when there is no constriction and $R_{c}=R_{m}$, to $s=1$, when the constriction is maximal and $R_{c}=0$.

\section{B. Exact numerical method}

Analytical formulas derived with the perturbative method are compared with the (exact) solution of the Euler-Lagrange equations computed numerically. The Euler-Lagrange equations do not have an analytical solution, in general, but can be solved numerically and different methods have been developed to solve them. As we did in previous works [19-21], we use here the methodology proposed in Refs. [31-32], and apply it to axisymmetric shapes subject to equatorial constriction stress with either polar radius $R_{m}$, enclosed volume $V$, or membrane area $A$ maintained constant. This methodology for axisymmetric shapes involves a different parametrization of the generating curve. The generating curve is parametrized in terms of the angle with the symmetry axis as a function of the generating curve length. The Euler-Lagrange equation gives the differential equations, which allows obtaining the angle with the symmetry axis along the generating curve. Conditions at the poles and constant volume or constant area conditions set boundary conditions to the differential equations. Linear tensions appear as matching conditions at 

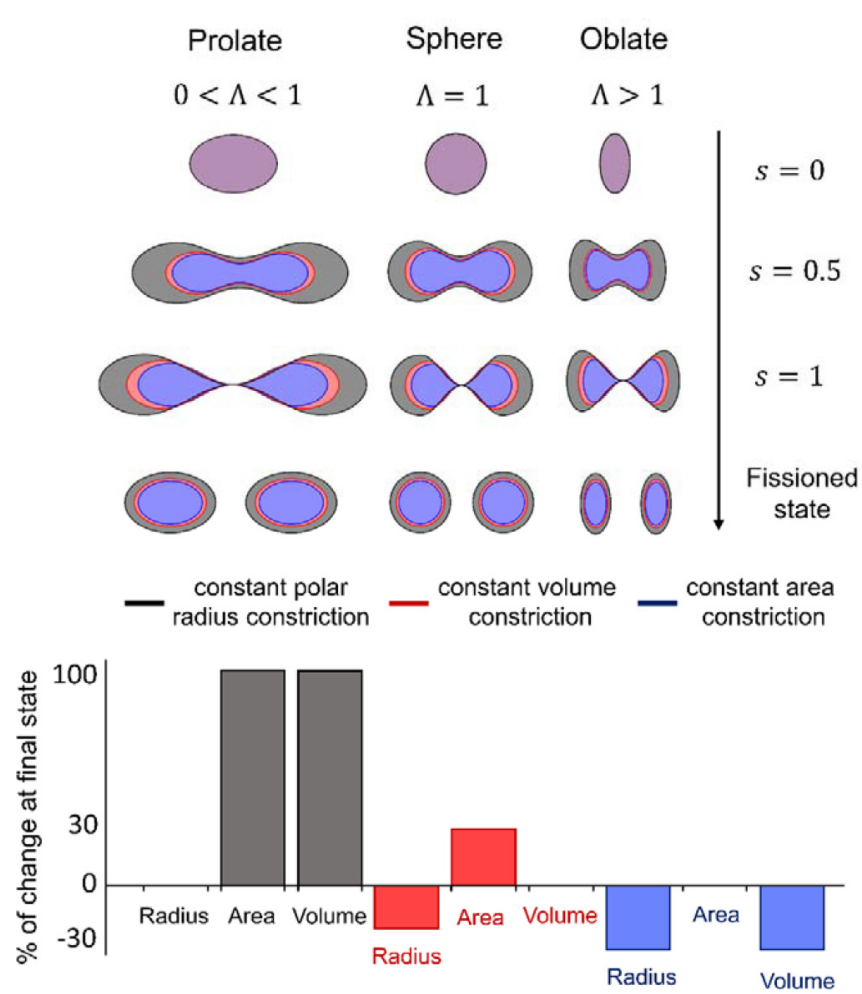

FIG. 3. Constraints to the constriction pathway. Upper panel: Model shapes for prolate, spherical, and oblate cells at different constriction stages (at $s=0$ : initially unconstricted vesicle; at $s=$ 0.5 : midpoint in the constriction process; at $s=1$ : maximum constriction; and at the finally fissioned state). Three different constraints to the constriction pathway are considered: constant polar radius (black shadow), constant volume constriction (red shadow), and constant area constriction (blue shadow). Lower panel: Constriction at constant polar radius requires an increase of $100 \%$ in membrane area and volume (black columns); constriction at constant volume requires a decrease in polar radius of around $20 \%$ and an increase in membrane area of around $26 \%$ (red columns); and constriction at constant area requires a decrease in both polar radius and volume of around $30 \%$ (blue columns). The cell shapes and the values in this figure were computed using the numerical solution.

the intermediate points where they are applied. (For a detailed description of the numerical procedure followed see the Supplemental Material of Ref. [21]). The MATHEMATICA numerical code used for the present paper is available in the Supplemental Material [53].

\section{Constraints to the constriction pathway}

Constant polar radius constriction. In a previous work [21], we explored the case of symmetric cell division with constant polar radius $R_{m}$ (i.e., constant maximum transversal radius; see Fig. 2). In this case, the shape of the lobes remains equal to the initial prolate, spherical, or oblate configuration at all stages of constriction while the cell area and volume are doubled upon division (Fig. 3, black shadowed shapes and bar charts).

Constant volume or area constriction. In this work, instead of constant polar radius constriction, we address constant volume or constant area constriction. Constriction at constant volume may describe dividing cells with intense membrane trafficking [31,32], which is known to play an important role in cytokinesis $[33,34]$. Constriction at constant area may describe dividing cells with low or inhibited membrane trafficking. In constant area constriction, a greater initial area is required to have the same final volume [19-21]. Heat shock has been shown to increase the area before division $[35,36]$ and to affect the gene expression of membrane trafficking molecules, but also those of signaling molecules [37].

There are several possibilities to define these constant area or constant volume constriction paths. In previous works [19-21], we proposed a redimensioning strategy that rescales the shape in a factor $\lambda(s)$ as $\kappa \rightarrow \kappa, R_{m} \rightarrow \lambda R_{m}, C_{0} \rightarrow$ $C_{0} / \lambda, A \rightarrow \lambda^{2} A, V \rightarrow \lambda^{3} V, \Sigma \rightarrow \Sigma / \lambda^{2}$, and $\Delta p \rightarrow \Delta p / \lambda^{3}$ [with $\lambda_{A}(s)$ at constant area constriction and with $\lambda_{V}(s)$ at constant volume constriction]. Note that this rescaling constriction path also keeps constant the dimensionless products $C_{0} R_{m}, \Sigma R_{m}^{2}$, and $\Delta p R_{m}^{3}$ (and thus $\Lambda$ ) during constriction. Although this simplifies the computations, it could be not very realistic. We explore here a more lifelike constriction path, where the parameters $C_{0}, \Sigma$, and $\Delta p$ remain constant along the constriction pathway (instead of the products $C_{0} R_{m}, \Sigma R_{m}^{2}$, and $\Delta p R_{m}^{3}$ ). To do that, we have first to determine the variation of the polar radius during constriction, $R_{m, A}(s)$ or $R_{m, V}(s)$, which also keeps constant either the cell membrane area $A$ or the volume enclosed by cell $V$, respectively. Therefore, we solve, respectively, the algebraic equations $A\left(R_{m}, s\right)=$ $A\left(R_{0}, 0\right)$ or $V\left(R_{m}, s\right)=V\left(R_{0}, 0\right)$, with $R_{0}$ the polar radius of the initial (unconstricted) cell [21]. Thus, the quantity $\Lambda$ of Eq. (6), which determines the shape of the cell lobes, mildly varies along the constriction process due to the variations of $R_{m, A V}(s)$ (for details, see Appendix A 3). The model predicts in general shape conservation; thus, oblate, spherical, or prolate mother cells divide, respectively, into two oblate, two spherical, or two prolate daughter cells (see Fig. 3). At constant volume constriction, each of two daughter parts reduces its polar radius by $21 \%$ and its membrane area by $37 \%$. This only requires an increase in the total area of $26 \%$. Instead, if the constriction process occurs keeping the membrane area constant, each of the two daughter parts reduces its polar radius by $29 \%$ and its volume by $64 \%$ upon constriction. This requires a reduction in the total volume of $29 \%$. These results are summarized in Fig. 3 (for further details, see Figs. 6 and 7 of the Appendix).

\section{Constriction force}

The constriction force, exerted as a constriction ring at the cell equator, is obtained from the variation of the total energy with respect to the constriction radius $R_{c}$. Under the constant polar radius $R_{m}$ condition, the constriction $F_{c, R_{m}}$ is obtained as $F_{c, R_{m}} \equiv-d E_{T} / d R_{c}=-\left(\partial E_{T} / \partial s\right)\left(\partial s / \partial R_{c}\right)=$ $1 / R_{m}\left(\partial E_{T} / \partial s\right)$, where we have used $s=1-R_{c} / R_{m}$. The constriction force scales inversely proportional to $R_{m}$; i.e., smaller cells are harder to constrict. This quantity was previously derived by us (both analytically and numerically) in Ref. [21]. Under the new constraints considered in this work, the constriction forces at constant area $F_{c, A}$ or at constant 
(a) $C_{0} R_{m}=-0.8$

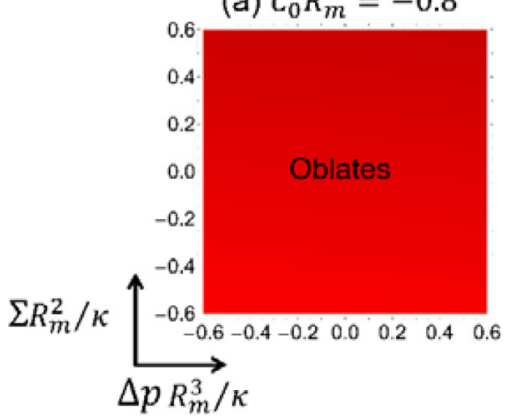

(d) $C_{0} R_{m}=0.8$

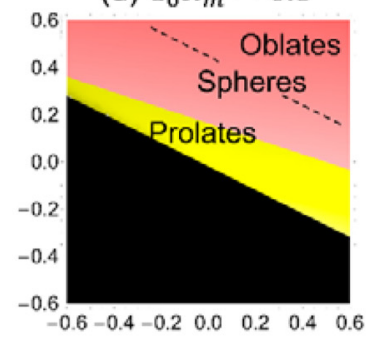

(b) $C_{0} R_{m}=-0.3$

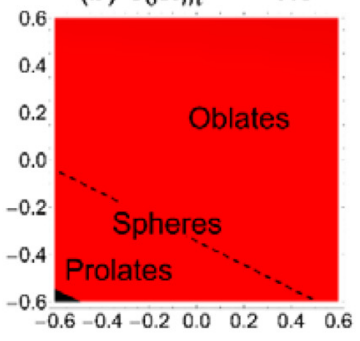

(e) $C_{0} R_{m}=1.3$

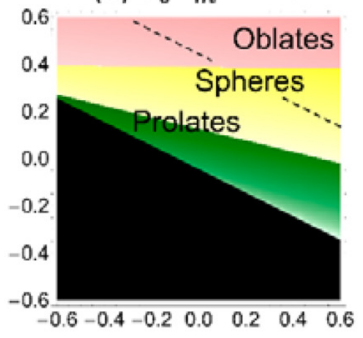

(c) $C_{0} R_{m}=0.3$

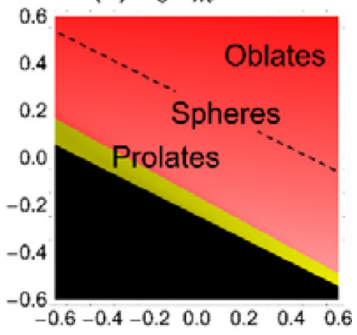

$$
\text { (f) } C_{0} R_{m}=1.8
$$

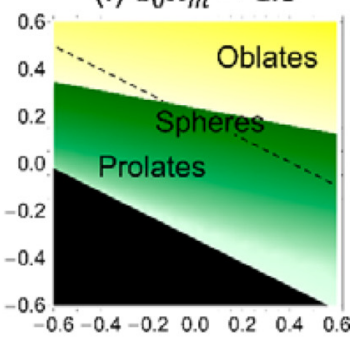

(g)

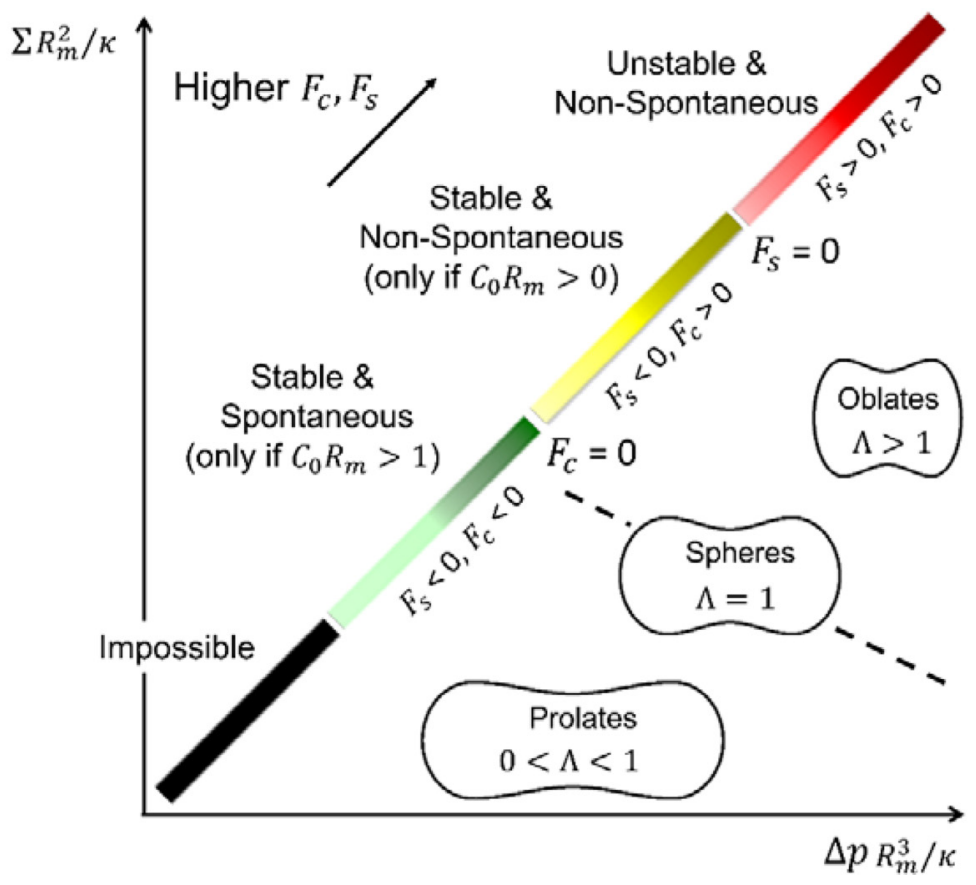

FIG. 4. Stabilization and constriction forces for different scenarios. Stabilization and constriction forces, $F_{s}$ and $F_{c}$, at the beginning of constriction $(s=0.2)$ as a function of the adimensionalized tension $\Sigma R_{m}^{2} / \kappa(y$ axis $)$ and the adimensionalized pressure $\Delta p R_{m}^{3} / \kappa(x$ axis) for different values of the adimensionalized spontaneous curvature: $C_{0} R_{m}=-0.8$ (a), $C_{0} R_{m}=-0.3$ (b), $C_{0} R_{m}=0.3$ (c), $C_{0} R_{m}=0.8$ (d), $C_{0} R_{m}=1.3(\mathrm{e})$, and $C_{0} R_{m}=1.8$ (f). Conditions predicting unstable and nonspontaneous constrictions are shaded in red; conditions predicting stable but nonspontaneous constrictions are shaded in yellow; conditions predicting stable and spontaneous constrictions are shaded in green; and conditions under which equatorial constriction is impossible (imaginary analytical results and no numerical solution) are shaded in black. Stable symmetric constriction requires positive spontaneous curvature [(c)-(f)] while spontaneous symmetric constriction requires that the spontaneous curvature be higher than the characteristic radius of the cell [(e)-(f)]. Panel (g) graphically summarizes the results showing the different regimes, their locations, and separation conditions; cell shapes of three illustrative cases are represented. High surface tensions $\Sigma$ and high external pressures $\Delta p$ give rise to oblate vesicles, which require large constriction and stabilization forces. In contrast, if $\Sigma$ and $\Delta p$ are low, our model predicts prolate shapes requiring smaller forces. Dashed lines correspond to spherical vesicles [curves with $\Lambda=1$, Eq. (6)], the case that separates prolates $(0<\Lambda<1)$ and oblates $(\Lambda>1)$ (note that the curve with $\Lambda=1$ for $C_{0} R_{m}=-0.8$ is not in the region shown). The stabilization force $F_{s}$ and the constriction force $F_{c}$ were determined assuming constant volume constriction, but the same conclusions apply for constant area constriction. This figure represents the results obtained with the analytical solutions, verified by comparison with the numerical solutions in a representative grid in the plotted ranges. 
volume $F_{c, V}$ are determined as

$$
\begin{aligned}
F_{c . A V}(s)= & \frac{1}{R_{m}} \frac{\partial E_{T}}{\partial s}+\frac{1}{R_{m}^{2}} \frac{\partial R_{m}}{\partial s} \\
& \times\left(\widetilde{C_{0}} \frac{\partial E_{T}}{\partial \widetilde{C}_{0}}+2 \tilde{\Sigma} \frac{\partial E_{T}}{\partial \tilde{\Sigma}}+3 \widetilde{\Delta p} \frac{\partial E_{T}}{\partial \widetilde{\Delta p}}\right),
\end{aligned}
$$

where $\widetilde{C_{0}}=C_{0} R_{m}, \tilde{\Sigma}=\Sigma R_{m}^{2}, \widetilde{\Delta p}=\Delta p R_{m}^{3}$, and $R_{m}=$ $R_{m, A}(s)$ or $R_{m}=R_{m, V}(s)$ is a function of the constriction state $s$ given by the assumed constraint of constant area or constant volume constriction, respectively. The constriction process is spontaneous for negative constriction force, $F_{c} \leqslant 0$, since this condition implies a decrease in the total energy as constriction progresses.

\section{E. Stabilization force}

The stability of symmetric constriction against longitudinal asymmetries can be addressed by comparing the energy of the symmetrical cell with equal right and left lobes with an asymmetrical cell in which the constriction ring is displaced a small distance $\Delta x$ from midcell [see Fig. 1(b)]. In the asymmetrical case, the polar radius $R_{m}$ (and consequently the constriction parameter $s$ ) is slightly different in each one of the lobes. At constant area (or volume) constriction, the transition between the symmetric and the asymmetric shape is assumed to occur by keeping the area (or volume) constant. For small displacements $\Delta x$, we can write

$$
E_{\text {asym }, A V}(s)=E_{\text {sym }, A V}(s)+k_{A V}\left(\frac{\Delta x}{R_{m, A V}}\right)^{2},
$$

where $k_{A V}$ is the stability coefficients with units of energy $\left(k_{A}\right.$ for constant area and $k_{V}$ for constant volume) [19,20]. Symmetric constriction is stable for positive stability coefficients, as then the energy of the symmetric configuration is smaller than for the asymmetric one. In contrast, negative stability coefficients predict unstable symmetric shapes against longitudinal deformations. The stabilization forces at constant area $F_{s, A}$ or constant volume constriction $F_{s, V}$ are obtained from Eq. (8) as

$$
F_{s, A V}(s)=-\left.\frac{\partial E_{\mathrm{asym}}}{\partial \Delta x}\right|_{A V}=-2 k_{A V} \frac{\Delta x}{R_{m, A V}^{2}} .
$$

This definition implies that symmetric constriction is stable for negative stabilization force, $F_{s} \leqslant 0$, which scales inversely proportional to $R_{m}^{2}$; i.e., smaller cells require higher stabilization forces for symmetric constriction. The deduction of the stability coefficients together with their analytical approximate solutions can be found in Appendix A4. Recall that $R_{m, A V}$ in Eqs. (8) and (9) is a function of the constriction state $s$, a function given by the assumed constraint of constant area or constant volume constriction.

\section{RESULTS}

Our minimal model of cell constriction includes the essential mechanical ingredients involved in cytokinesis, i.e., flexibility of the shell enclosing the cell, possible geometrical constraints, and driving forces leading to equatorial constriction and stability. In this setting, we analyze the mechanical requirements for stable symmetric constriction in different conditions. In particular, the constriction forces required having constriction, and the stabilization forces required keeping the constriction symmetric. The results obtained indicate that the main magnitude is the spontaneous curvature, $C_{0}$, which separates three regions (see Fig. 4):

(1) Negative spontaneous curvature, $C_{0}<0$ : Unstable constriction. For negative spontaneous curvature, the constriction is nonspontaneous and unstable irrespective of surface tension $\Sigma$ and external pressure $\Delta p$ [see Figs. 4(a) and 4(b)]. Cells with a negative spontaneous curvature are prone to build up in a concave configuration. In constricting cells, although the cell shape in the central constricting region is concave as seen from the cell exterior, the cell is convex in the other cell regions. Thus, membranes with negative spontaneous curvature require additional mechanisms to generate and stabilize symmetric constriction.

(2) Moderately positive spontaneous curvature, $0 \leqslant C_{0}<$ $1 / R_{m}$ : Stable constriction is possible. Constriction is always nonspontaneous in this case, i.e., when the positive spontaneous curvature is smaller than the inverse of the characteristic radius of the cell. However, symmetric constriction may be stable for a narrow range of surface tension and external pressure, where cells do not need symmetrization mechanism, only constriction mechanisms. [This range is shaded in yellow in the (c) and (d) panels of Fig. 4.]

(3) Considerably positive spontaneous curvature, $C_{0} \geqslant$ $1 / R_{m}$ : Spontaneous and stable constriction is possible. If the spontaneous curvature is higher than the inverse of the cell size, there exists a broad region of values of surface tension $\Sigma$ and external pressure $\Delta p$ giving rise to spontaneous and stable symmetric constriction [see region shaded in green in Figs. 4(e) and 4(f)]. This region requires neither constriction nor stabilization forces to generate and stabilize the symmetric constriction.

Therefore, stable symmetric constriction without centering mechanisms (i.e., zero stabilization force) requires cells with a globally positive spontaneous curvature that decreases the bending energy. In order to be additionally spontaneous (i.e., zero constriction force), the cell requires spontaneous curvature higher than its characteristic inverse size, $C_{0} \geqslant 1 / R_{m}$. The later spontaneity condition is found to be stricter, and spontaneous constriction is always stable, as shown by the region configuration in Fig. 4.

Reducing either the surface tension or the external pressure increases the stability and spontaneity of constriction, as shown in Fig. 4. To symmetrically constrict in a stable and spontaneous way, cells subjected to high external pressures $(\Delta p>0)$ require negative surface tensions $(\Sigma<0)$, much higher in absolute value than cells immersed in an iso- or hypotonic media ( $\Delta p \leqslant 0$ ) [panels (c)-(f) of Fig. 4]. This result means that negative surface tensions $\Sigma$ may counterbalance high positive pressures $\Delta p$. Likewise, negative pressures may counterbalance high positive membrane tensions. However, below a critical threshold, $\Lambda \leqslant 0$, which corresponds to strong negatives values of both $\Sigma$ and $\Delta p$ (regions shaded in black in Fig. 4), symmetric constriction is no longer possible. As we approach the limit $\Lambda \rightarrow 0$, our model predicts very elongated prolate (rodlike) cells, for which the symmetric mode of equatorial constriction is impossible since these cells cannot fix a single circumferential furrow at a well-defined equator. 

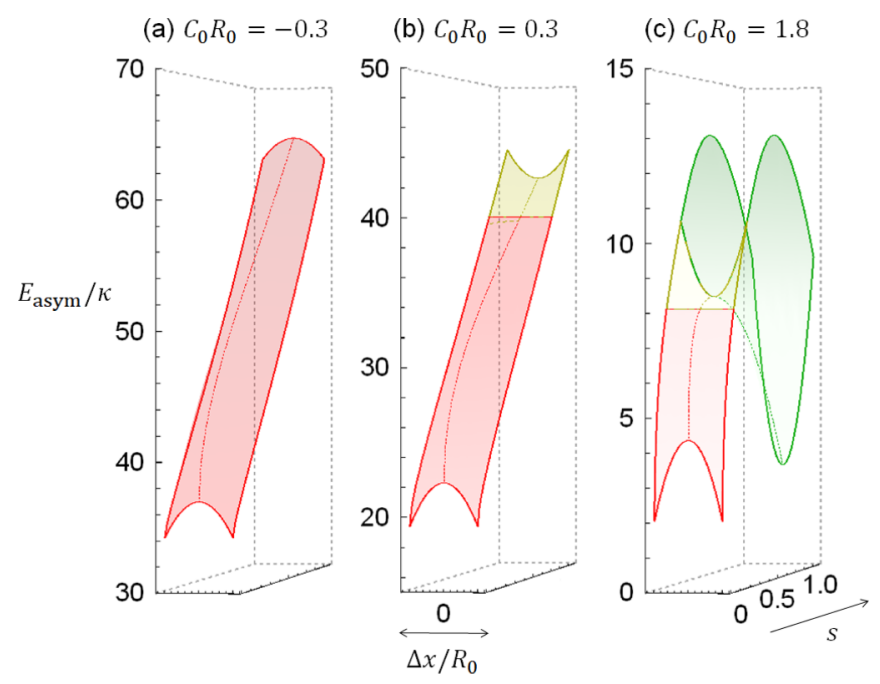

FIG. 5. Energy landscape during cell constriction for different scenarios. Energy of the asymmetric configuration as a function of the constriction stage $s$ and the displacement of the constriction ring from the middle point $\Delta x / R_{0}$ for $\Sigma R_{0}^{2} / \kappa=\Delta p R_{0}^{3} / \kappa=0.25$ and for $C_{0} R_{0}=-0.3$ (a), 0.3 (b), and 1.8 (c), with $R_{0}$ the initial maximum radius. Dotted line represents the symmetric constriction pathway. Unstable, stable, and spontaneous regions are shaded, respectively, in red, yellow, and green. Membranes characterized by negatives values of $C_{0}$ (a) present unstable (convex profile) and nonspontaneous constriction (uphill energy) during the whole process. If, instead, $C_{0}$ is positive but lower than the characteristic inverse cell size (b), the constriction is nonspontaneous (uphill energy) but reaches stability at some point of the division (concave profile). Finally, when $C_{0}$ is higher than the characteristic inverse cell size (c), constriction starts as a nonspontaneous and unstable process but becomes stable (concave profile) and spontaneous (downhill energy) at final stages. These results were determined assuming constant volume constriction, but the same conclusions apply for constant area constriction. This figure represents the results obtained with the analytical solutions, verified by comparison with the numerical solutions in a representative grid in the plotted ranges.

In general, prolate (rodlike) shaped cells require lower stabilization and constriction forces than oblate (disklike) cells. This result is related to the previously described effects of the pressure and tension, as lower values of surface tension and external pressure tend to give prolate (rodlike) cell shapes, which are found to require lower stabilization and constriction forces. Conversely, higher values of surface tension and external pressure give rise to oblate (disklike) cells, which require strong constriction and stabilization forces to divide symmetrically (see Fig. 4).

Stability and spontaneity of symmetric constriction are found to increase as constriction progresses, reducing the required stabilization and constriction forces, as shown in Fig. 5. Nonspontaneous constrictions are uphill processes, characterized by an increase of energy as the constriction advances, and therefore require a constriction force to occur. This can be seen in Fig. 5 as an increase of energy in the direction of the constriction stage $s$ coordinate for the nonspontaneous regions (red and yellow regions). Note also that progress in constriction implies a decrease in the slope (related to constriction force), which in some cases even becomes negative, implying a transition from nonspontaneous (red and yellow region) to spontaneous constriction (green region). Stable symmetric constriction entails that symmetric constriction is an energy minimum, and therefore the energy increases when the asymmetry $\Delta x$ goes out of its zero value, giving a concave energy profile in the $\Delta x$ direction for the stable cases (yellow and green regions of Fig. 5). Unstable symmetric constriction corresponds to maxima, and convex profiles appear in the $\Delta x$ direction (red regions of Fig. 5). Figure 5 illustrates that as symmetric constriction progresses, it becomes more stable, in addition to more spontaneous; i.e., both the required stabilization and constriction forces are reduced along the constriction pathway.

Membranes characterized by negatives values of $C_{0}$ present unstable and nonspontaneous constriction during the whole process [Fig. 5(a)]. On the other hand, if $C_{0}$ is positive but lower than the characteristic inverse cell size, the constriction is nonspontaneous but reaches the stability at some point of the division [Fig. 5(b)]. Finally, when $C_{0}$ is higher than the characteristic inverse cell size, constriction starts as a nonspontaneous and unstable process but becomes stable and spontaneous at the final stages [Fig. 5(c)]. The constriction stages where the transitions between the regions occur depends on the values of the membrane surface tension and external pressure. Note also that the energy of the system decreases as the spontaneous curvature increases.

We also note that for constant volume or constant area constriction, $R_{m}$ decreases as constriction advances (see Figs. 6 and 7 of the Appendix). This implies that the product $C_{0} R_{m}$ also decreases during constriction, and therefore it could change from one region of behavior to another. In practice, this only happens to values close to the limits of the region (as for example $C_{0} R_{0}=1.3$ ). In particular, it is not the case for the values represented in Fig. 5.

\section{DISCUSSION}

We found that mechanical stability of symmetric constriction without centering cytokinetic forces necessarily requires cell shapes defined by positive spontaneous curvature (prolate-like) at sufficiently high external pressure and low membrane tension [see Figs. 4(e) and 4(f)], a situation found in some elongated eukaryote cells [39]. Otherwise, additional stabilization mechanisms should be incorporated into the constriction system. Amazingly, symmetric constriction is shown to occur spontaneously for spontaneous curvatures above a threshold determined by the cell size $\left(C_{0} \geqslant 1 / R_{m}\right)$. This circumstance is not usual in modern cells endowed with specific cytokinetic machinery, but it could have been exploited by primitive protocells to undergo spontaneous division [40]. Furthermore, our results show that a lowering in membrane tension, due to membrane-targeted lipid biogenesis or directed cortical flows, for instance, leads to an increase of stability and spontaneity of cellular constriction. A reduction of external pressure also gives rise to a similar consequence. This implies that prolate-shaped cells (cylinders) favored by low pressure and surface tension, in general, require lower constriction and stabilization forces to undergo division.

These general results are useful for the study of a wide range of animal cells, as they are independent of the detailed 
mechanism, and give the force requirements that have to be verified by the combined action of the different mechanism in action in this cell type. This is particularly useful because mechanical properties of the cortex present a huge degree of variability between cell types, and may change during the cell cycle [18]. The osmotic pressure difference in animal cells is in the range of $0.1-1 \mathrm{kPa}$, cortical tension in the range of $40-4000 \mathrm{pN} / \mu \mathrm{m}$, and cortex thickness in the range of $50 \mathrm{~nm}$ to $1 \mu \mathrm{m}$ [18]. The Young's modulus of the cell cortex is on the order of $100 \mathrm{kPa}$ [22]. These latter values imply effective bending rigidities in the range of $10^{3}-10^{7} k_{B} T$, which dominate the mechanics of the cortical layer, as they are much greater than the typical bending rigidities of the lipid bilayer $\left(10-200 k_{B} T\right)$. These parameter values lead to similar energy contributions of the pressure, tension, and bending contributions, and to stabilization and constriction forces on the order of tenths of nanonewtons. This constriction force value is in accordance with measurements [41] and can be generated by polymerization and depolymerization in actin filament bundles [42].

Alternatively, in the simplest case of cell-sized lipid vesicles $\left(R_{0} \approx 1-10 \mu \mathrm{m}\right)$, with a single lipid bilayer as the flexible membrane $\left(\kappa \approx 20-200 k_{B} T\right)$, the required constriction forces are smaller, in the piconewton range, $F_{c} \approx \kappa / R \approx$ $0.01-1 \mathrm{pN}$. Under adequate conditions of excess area, or reduced volume, regulated by osmotic stresses, asymmetric budding occurs spontaneously in cell-size vesicles without constriction machinery [43]. This indicates the chief role of membrane tension and external pressure in spontaneous constriction mechanisms leading to facilitated cell scission. Indeed, well known is the mechanical link that exists between the spontaneous curvature of the lipid bilayer and the phenomenon of spontaneous fission observed in model vesicles [44]. Those phenomena observed in artificial vesicles have attracted much theoretical interest [45-47]. Our minimal model enables predicting conditions for stability and spontaneity of the constriction process, and its general results are consistent with the experimental observations [41,48-50].

Relevant extensions of the present minimal model include studying mechanical conditions to stabilize asymmetric constrictions found in some cell types, which will help to understand this open question [17]. Another extension of our model would entail the inclusion of the cell wall effects to determine minimal mechanical requirements in prokaryotes and plant cell division [15].

\section{ACKNOWLEDGMENTS}

All authors acknowledge Alan Siegel, Harold C. Smith, and Andrew Wilde for providing permission to use their images in Fig. 1(a). E.B.H. acknowledges financial support from Ministerio de Educación, Cultura, y Deporte (MECD, Spain) under FPU Grant No. 13/02826. F.M. acknowledges financial support from Ministerio de Economía y Competitividad (MINECO, Spain) and European Regional Development Fund (ERDF) under Grant No. FIS201570339-C21-R, and from Comunidad de Madrid (CAM, Spain) under Grant No. S2013/MIT-2807. F.J.C.G. acknowledges financial support from Ministerio de Economía y Competitividad
(MINECO, Spain) and from European Regional Development Fund (ERDF) under Grant No. FIS2015-67745-R.

E.B.H. proposed the research. F.J.C.G. coordinated the research. E.B.H. and F.J.C.G. developed the analytical and numerical techniques. E.B.H. performed the analytical and numerical computations. F.M. prepared Fig. 1(b). E.B.H. prepared Figs. 2-5 and wrote the first version of the Appendix. E.B.H., F.M., and F.J.C.G. wrote the paper.

\section{APPENDIX}

\section{Equilibrium condition: Generalized young-laplace equation as constitutive relationship relating systemic constraints and cellular shape}

In our minimal model of a deformable cell depicted as a closed vesicle of membrane area $A$ and enclosed volume $V$, under the net pressure difference $\Delta p=p_{\text {out }}-p_{\text {in }}$ between the outer medium and the cell interior, and the tensional action of the surface tension $\Sigma$, the total elastic energy is written in the canonical Canham-Helfrich form as [28]

$E_{T}=\int_{\Omega}\left[\frac{\kappa}{2}\left(2 H-C_{0}\right)^{2}+\kappa_{G} K+\Sigma\right] d A+\int_{\Omega} \Delta p d V$,

where $\Omega$ is the closed surface that delimits the membrane vesicle, $H$ is the mean curvature, $K$ the Gaussian curvature, $d A$ denotes the element of area, and $d V$ the volume counterpart. The parameters $\kappa$ and $\kappa_{G}$ are the bending modulus and the Gaussian bending rigidity, which represent the strength of the membrane modes of curvature associated with $H$ and $K$, respectively; $C_{0}$ is the spontaneous curvature of the membrane, which represents the tendency of the membrane to curve in the equilibrium state [28], usually due to the compositional asymmetry between the inner and the outer sides [29]. Note that the Gaussian term $\int_{\Omega} \kappa_{G} K d A$ is omitted in Eq. (A1). This is because this integral is constant for surfaces with the same topology (Gauss-Bonnet theorem [28]). Since the constriction process in a vesicle does not change its topology, and only involves shapes that are topologically equivalent to a sphere (no holes), the contribution of the Gaussian curvature can be ignored because it remains constant independently of the size and shape of the vesicle. For the final fissioned state, in which the vesicle splits into two separated daughters, the Gaussian contribution should be considered.

To determine which generic shape has the lower energy for given values of the mechanical constraints, the first variation of the energy is calculated from Eq. (A1). For a closed membrane vesicle, the equilibrium cell-shape equation is [51,52]

$$
\begin{aligned}
& \Delta p+2 \Sigma H-\kappa\left(2 H-C_{0}\right)\left(2 H^{2}-2 K+C_{0} H\right)-2 \kappa \nabla^{2} H \\
& \quad=0,
\end{aligned}
$$

where $\nabla^{2}$ is the Laplace-Beltrami operator. This nonlinear partial differential equation is extremely complicated to solve. It represents the local force balance at the vesicle membrane, and apart from the pressure difference and the membrane tension, it accounts for the local stress of curvature.

For the special case of a sphere of constant radius $R$, the curvatures are $H=1 / R$ and $K=1 / R^{2}$, so the shape equation has the analytical solution [28]

$$
\Delta p R^{3}+2 \Sigma R^{2}+\kappa\left(C_{0} R-2\right) C_{0} R=0,
$$


which is fulfilled under the geometrical condition $\nabla^{2} H=$ 0 , which describes the constant curvature of the sphere. Amazingly, if no bending rigidity is considered $(\kappa=0)$, the relationship in Eq. (A3) reduces to the well-known YoungLaplace equation of a soap bubble, $\Delta p=-2 \Sigma / R$, which relates the radius of the bubble with the Laplace pressure through the surface tension. Thus, positive membrane tension leads to a negative value of the Laplace pressure, as defined by the setting in Eq. (A1).

In a previous work [21], we derived the following generalized Young-Laplace relationship for ellipsoidal spheroids with revolution symmetry

$\Delta p R_{m}^{3}+2 \Sigma R_{m}^{2}+\kappa\left(C_{0} R_{m}-2\right) C_{0} R_{m}+\kappa(1-\Lambda)=0$,

where $R_{m}$ is the polar radius of the spheroid and $\Lambda$ is the shape parameter, which takes values $0<\Lambda<1$ for prolate spheroidal shapes, $\Lambda>1$ for oblate ones, and reduces to $\Lambda=1$ for the sphere (see Fig. 3). This expression leads to the definition of $\Lambda$ shown in Eq. (2). For small departure from the spherical shape, the curvatures of spheroids may approach those of the sphere, and then $H \approx 1 / R$ and $K \approx 1 / R^{2}$. Thus, by comparison between Eqs. (A2) and (A4), one deduces the Laplacian-Beltrami term in Eq. (A2) for spheroids as $\nabla^{2} H=$ $(\Lambda-1) / 2 R_{m}^{3}$. The Laplacian of a scalar field measures the convexity, or stress of the field, as indicated by how much the value of the field differs from its average value taken over the surrounding points. Oblate shapes $(\Lambda>1)$ give positive values of $\nabla^{2} H$, which indicates locally concave scalar curvature fields. Conversely, prolate shapes $(0<\Lambda<1)$ give $\nabla^{2} H<0$, corresponding to locally convex scalar curvature fields.

\section{Differential geometry of spheroidal cells}

To describe the membrane surface of the vesicle, we use the Monge parametrization [28]: the position $\vec{r}$ on the surface has Cartesian coordinates and is defined by giving its height $h$ over some $x-y$ plane as

$$
\vec{r}=(x, y, h(x, y)) .
$$

After considering mathematical aspects of differential geometry, the mean curvature $H=\left(C_{1}+C_{2}\right) / 2$ is found to be given in terms of the height function by [28]

$$
H=\frac{C_{1}+C_{2}}{2}=\frac{\left(1+h_{x}^{2}\right) h_{y y}+\left(1+h_{y}^{2}\right) h_{x x}-2 h_{x} h_{y} h_{x y}}{2\left(1+h_{x}^{2}+h_{y}^{2}\right)^{3 / 2}},
$$

where the $x$ and $y$ subscripts represent partial derivatives with respect to this variable. The element of area on the surface and the element of volume enclosed by the surface area [28]

$$
d A=\sqrt{1+h_{x}^{2}+h_{y}^{2}} d x d y, d V=h d x d y .
$$

For the particular case of axisymmetric shapes with the axis of symmetry along the $x$ axis,

$$
h(x, y)= \pm \sqrt{R^{2}(x)-y^{2}},
$$

where $R(x)$ is the functional form describing the membrane profile in the $x-z$ plane (see Fig. 2). The signs + and parametrize, respectively, the upper half $\Omega_{+}$and the lower half $\Omega_{-}$of the surface. Using the parametrizations for $\Omega_{+}$the mean curvature of Eq. (A6) becomes

$$
H=\frac{C_{1}+C_{2}}{2}=\frac{1+R_{x}^{2}-R_{x x} R}{2\left(1+R_{x}^{2}\right)^{3 / 2} R} .
$$

The elements of area and volume [Eq. (A7)] are

$$
d A=\sqrt{\frac{1+R_{x}^{2}}{R^{2}-y^{2}}} d x d y, d V=\sqrt{R^{2}(x)-y^{2}} d x d y .
$$

If the surface is between $x_{i}$ and $x_{f}$, its total area is

$$
\begin{aligned}
A & =2 \int_{\Omega_{+}} d A=2 \int_{x_{i}}^{x_{f}} R \sqrt{1+R_{x}^{2}} \int_{-R(x)}^{R(x)} \frac{1}{\sqrt{R^{2}-y^{2}}} d y d x \\
& =2 \pi \int_{x_{i}}^{x_{f}} R \sqrt{1+R_{x}^{2}} d x .
\end{aligned}
$$

For the total volume enclosed by the surface we have

$$
\begin{aligned}
V & =2 \int_{\Omega_{+}} h(x, y) d x d y=2 \int_{\Omega_{+}} \sqrt{R^{2}(x)-y^{2}} d y d x \\
& =\pi \int_{x_{i}}^{x_{f}} R^{2} d x,
\end{aligned}
$$

where the factor 2 outside the integrals of Eqs. (A11) and (A12) comes from the symmetry between $\Omega_{+}$and $\Omega_{-}$, and we have integrated the $y$ variable.

Finally, the bending energy $E_{b}$ (ignoring the Gaussian term) for a surface of revolution is given by

$$
\begin{aligned}
E_{b}= & \frac{\kappa}{2} \int_{\Omega}\left(2 H-C_{0}\right)^{2} d A \\
= & \kappa \int_{x_{i}}^{x_{f}} \frac{\left[1+R_{x}^{2}-R_{x x} R-R C_{0}\left(1+R_{x}^{2}\right)^{3 / 2}\right]^{2}}{\left(1+R_{x}^{2}\right)^{5 / 2} R} \\
& \times \int_{-R(x)}^{R(x)} \frac{1}{\sqrt{R^{2}-y^{2}}} d y d x \\
= & \pi \kappa \int_{x_{i}}^{x_{f}} \frac{\left[1+R_{x}^{2}-R_{x x} R-R C_{0}\left(1+R_{x}^{2}\right)^{3 / 2}\right]^{2}}{\left(1+R_{x}^{2}\right)^{5 / 2} R} d x .
\end{aligned}
$$

Note that the results of Eqs. (A11)-(A13) are independent of the coordinate $y$, as expected for surfaces with rotational symmetry around $x$.

\section{Constriction at constant membrane area or at constant cell volume}

Instead of considering the polar radius $R_{m}$ constant, constrictions at constant area or at constant volume can be additionally addressed. There are several possibilities to define these constant area or constant volume constriction paths. In Refs. [19-21], we proposed a redimensioning strategy that rescales the shape in a factor $\lambda(s)$ as $R_{m} \rightarrow \lambda R_{m}, \kappa \rightarrow \kappa$, $E_{T} \rightarrow E_{T}, C_{0} \rightarrow C_{0} / \lambda, C_{1} \rightarrow C_{1} / \lambda, C_{2} \rightarrow C_{2} / \lambda, A \rightarrow \lambda^{2} A$, 

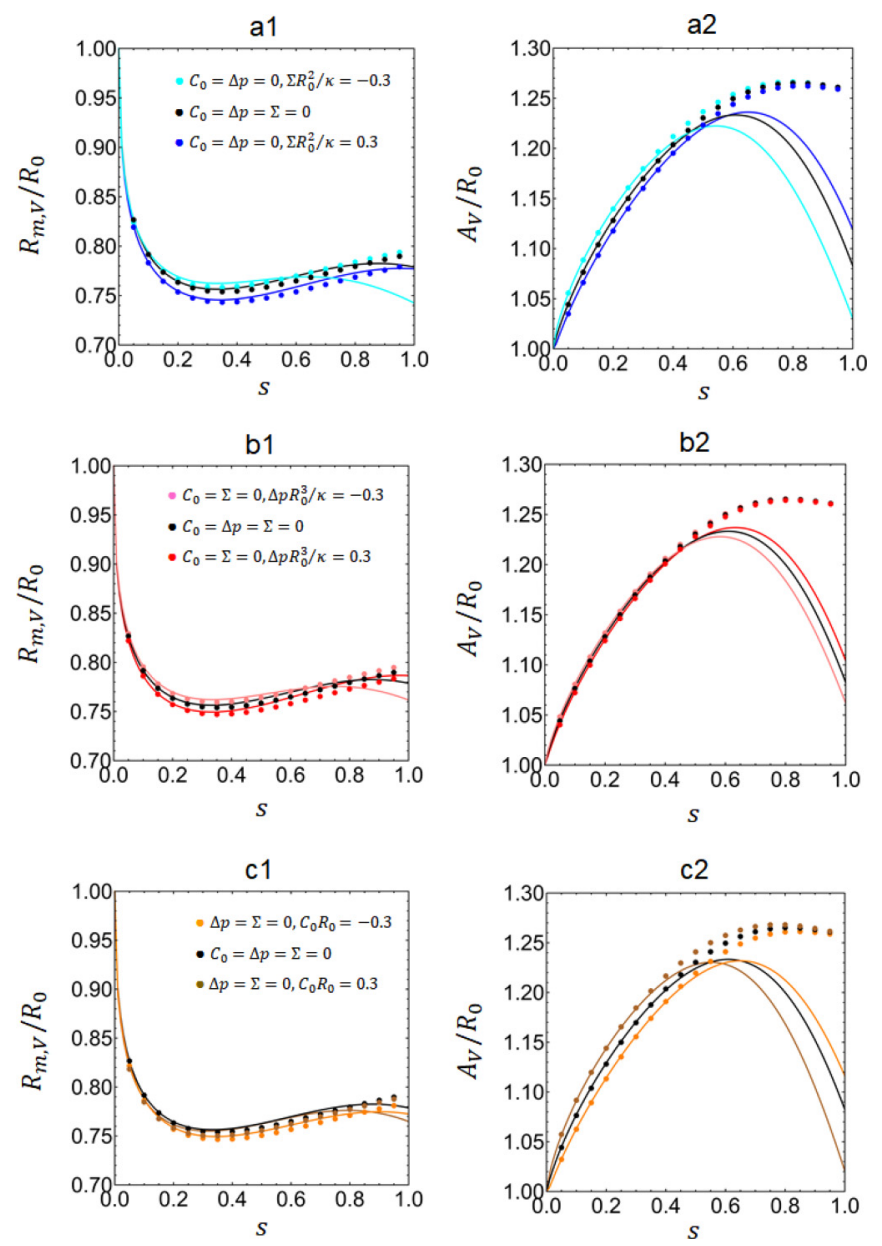

FIG. 6. Constant volume constriction. Decrease of the polar radius (left-hand column) and increase of membrane area (righthand column) along the constriction pathway maintaining a constant volume. Comparison between analytical and numerical results. (a) Influence of the surface tension $\Sigma$. (b) Influence of the osmotic pressure $\Delta p$. (c) Influence of the spontaneous curvature of the membrane $C_{0}$. Constriction process at constant volume requires a final decrease in polar radius of around $20 \%$ and a final increase in the membrane area of around $26 \%$, independently of $C_{0}, \Sigma$, and $\Delta p$.

$V \rightarrow \lambda^{3} V, \Sigma \rightarrow \Sigma / \lambda^{2}$, and $\Delta p \rightarrow \Delta p / \lambda^{3}$. This parameter corresponds to

$$
\lambda_{A}=\sqrt{\frac{A(s=0)}{A(s)}}
$$

for the constant area condition and to

$$
\lambda_{V}=\sqrt[3]{\frac{V(s=0)}{V(s)}}
$$

for the constant volume condition. $A(s=0)$ and $V(s=0)$ are, respectively, the membrane area and the volume enclosed by the initial spheroid. This rescaling method imposes shape invariance since it maintains the dimensionless products $C_{0} R_{m}, \Sigma R_{m}^{2}$, and $\Delta p R_{m}^{3}$ constant upon constriction [and then maintains $\Lambda$ invariant; see Eq. (3)]. Although this simplifies the computations, it could be not very realistic.
We explore here a more lifelike constriction path, where the parameters $C_{0}, \Sigma$, and $\Delta p$ remain constant along the constriction pathway (instead of products $C_{0} R_{m}, \Sigma R_{m}^{2}$, and $\left.\Delta p R_{m}^{3}\right)$. To do that, we compute the variation of the polar radius during constriction that keeps constant either the cell membrane area $A$ or the volume enclosed by the cell $V$, $R_{m, A V}(s)$, then allowing $\Lambda$ to vary in both cases along the constriction pathway. Therefore, we solve the respective algebraic equations, either $A\left(R_{m}, s\right)=A_{0}\left(R_{0}, s=0\right)$ at variable $V\left(R_{m}, s\right)$, or $V\left(R_{m}, s\right)=V_{0}\left(R_{0}, s=0\right)$ at variable $A\left(R_{m}, s\right)$, with $R_{0}$ being the polar radius of the mother cell (unconstricted configuration). The expressions of membrane area $A\left(R_{m}, s\right)$ and the cell volume $V\left(R_{m}, s\right)$ along the constriction pathway were previously derived for us (both analytically and numerically) in Ref. [21]. Except in the simple case when $C_{0}=\Sigma=\Delta p=0$, there is not an analytical solution of these equations, but they can be solved numerically. Equivalently, $R_{m, A V}(s)$ can be obtained by evaluating the following differential equations with the initial condition $R_{m, A V}(0)=R_{0}$ :

$$
\frac{d R_{m, A}}{d s}=-\frac{\partial A / \partial s}{\partial A / \partial R_{m}}, \frac{d R_{m, V}}{d s}=-\frac{\partial V / \partial s}{\partial V / \partial R_{m}} .
$$

Consequently, the quantity $\Lambda$ of Eq. (3), which determines the shape of the cell lobes, mildly varies along the constriction process due to the variations of $R_{m, A V}$. The model predicts in general shape conservation. Thus, oblate, spherical, or prolate mother cells divide, respectively, into two oblate, two spherical, or two prolate daughter cells (see Fig. 2).

Increase of area at constant volume. Constriction at constant volume requires a decrease in polar radius $R_{m, V}$ and an increase in the membrane area $A_{V}$ (see Fig. 6). There is a close agreement between the analytical and the numerical results for both quantities at low and intermediate regimes. At large constrictions, the analytical formulas do not describe the exact solution properly but significantly underestimate the increase of area. At final constriction, when $s \rightarrow 1$, the decrease in polar radius is

$$
\lim _{s \rightarrow 1} \frac{R_{m, V}}{R_{0}}=2^{-1 / 3} \approx 0.79,
$$

while the increase in membrane area is

$$
\lim _{s \rightarrow 1} \frac{A_{V}}{A_{0}}=2^{1 / 3} \approx 1.26 .
$$

Consequently, the constriction process at constant volume requires a final decrease in polar radius of around $20 \%$ and a final increase in the membrane area of around $26 \%$ independently of $C_{0}, \Sigma$, and $\Delta p$, which is in agreement with the numerical limits (see Fig. 6).

Constriction at constant volume may describe divided cells with intense membrane trafficking [31,32], which is known to play an important role in cytokinesis $[33,34]$.

Decrease of volume at constant area. Constriction at constant area requires a decrease in both the polar radius $R_{m, A}$ and the volume enclosed by the vesicle $V_{A}$ (see Fig. 7). As in constant volume constriction, the analytical results reproduce the exact solution in the range of low and intermediate constrictions, but fail at higher regimes. The decrease in both polar radius and volume enclosed on the vesicle at the final 

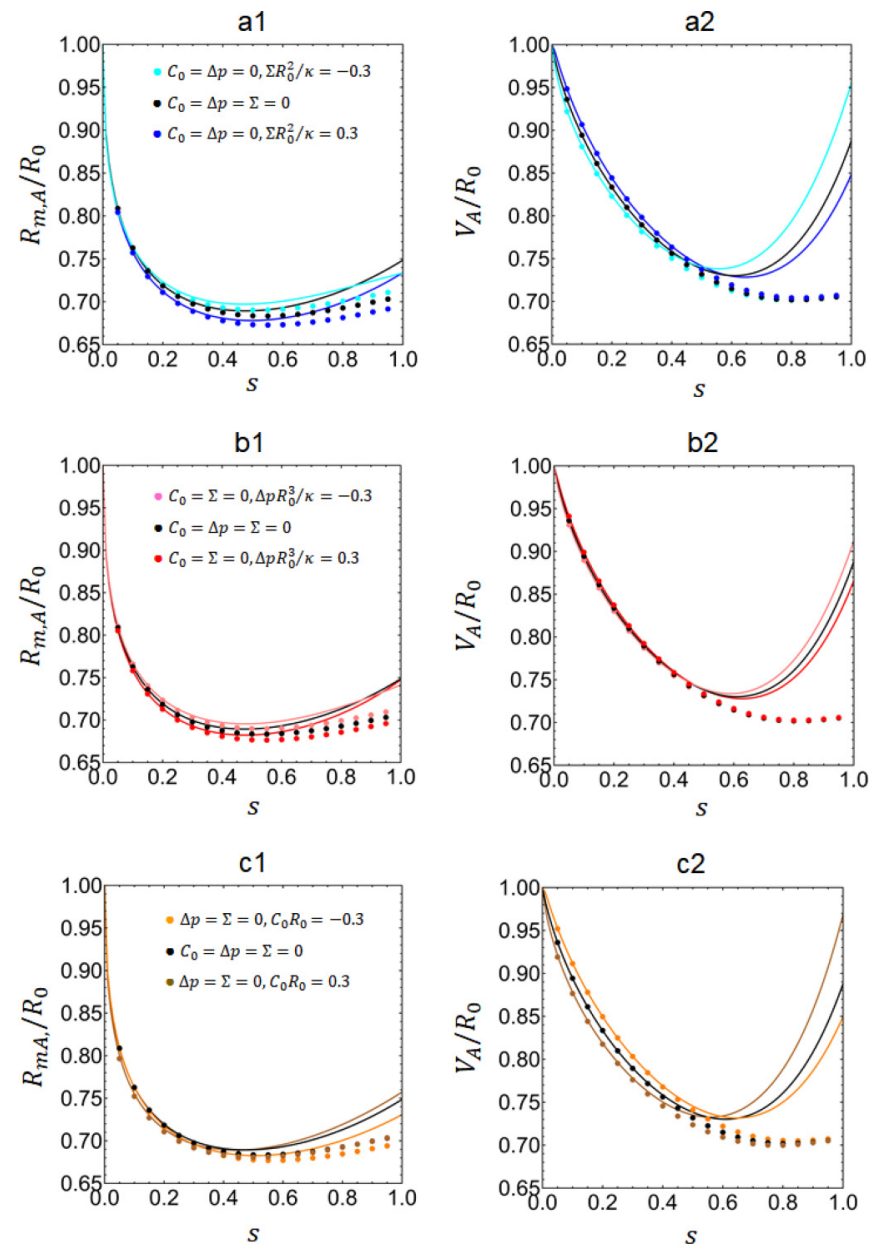

FIG. 7. Constant area constriction. Decrease of both polar radius (left-hand column) and the volume enclosed in the vesicle (right-hand column) during constant area constriction. Comparison between analytical and numerical results. (a) Influence of the surface tension $\Sigma$. (b) Influence of the osmotic pressure $\Delta p$. (c) Influence of the spontaneous curvature of the membrane $C_{0}$. Constriction process at constant area requires a final decrease in both polar radius and volume enclosed on the vesicle of around $30 \%$, independently of $C_{0}$, $\Sigma$, and $\Delta p$.

constriction state is

$$
\lim _{s \rightarrow 1} \frac{R_{m, A}}{R_{0}}=\lim _{s \rightarrow 1} \frac{V_{A}}{V_{0}}=2^{-1 / 2} \approx 0.71,
$$

implying that the constriction process at constant area requires a final decrease in both polar radius and volume enclosed by the vesicle of around $30 \%$ independently of $C_{0}, \Sigma$, and $\Delta p$, which corresponds with the numerical limit (see Fig. 7).

Constriction at constant area may describe divided cells with low or inhibited membrane trafficking. Moreover, in constant area constriction, a greater initial area is required to have the same final volume. Heat shock has been shown to increase the area before division $[35,36]$ and to affect the expression of genes of membrane trafficking molecules, but also of other genes such as those of signaling molecules [37].

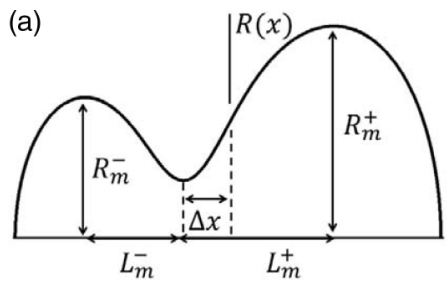

(b)

FIG. 8. Asymmetrically constricted vesicle. (a) Asymmetric profile after displacing the constriction ring a length $\Delta x$ from the middle point. (b) Surface obtained from the revolution around the $x$ axis.

\section{Stability of symmetric constriction: Stability coefficients}

The stability of the symmetric constriction can be addressed by introducing small changes to the symmetric shape and comparing its energy with the original one. In order to perform this computation, the constriction ring is displaced a length $\Delta x$ from the middle point giving an asymmetric shape in which one of the lobes is greater than the other (see Fig. 8).

We study here the cases in which the transition between the symmetric and the asymmetric shape takes place by keeping the volume enclosed in the vesicle or its membrane area constant. The energy of this new shape can be expressed as

$$
E_{\mathrm{asym}, V A}(s)=E_{\mathrm{sym}}(s)+k_{V, A}\left(\frac{\Delta x}{R_{m, A V}}\right)^{2},
$$

where $k_{V, A}$ are the stability coefficients with units of energy ( $k_{V}$ for constant volume and $k_{A}$ for constant area), and $R_{m, V A}$ is the polar radius. Symmetric constriction will be stable when the stability coefficients are positive, because, in this case, the energy of the symmetric configuration is smaller than the asymmetric one. In contrast, negative stability coefficients predict unstable symmetric shapes against longitudinal deformations. In terms of the first and second derivatives of $E_{T}$, $L_{T}=L_{m}+L_{p}$, and $V$ (for constant volume constriction), or $A$ (for constant area constriction) with respect $R_{m}$ (see next section for a detailed deduction), $k_{V, A}$ take the form

$$
\begin{aligned}
& k_{V}=\frac{R_{m, V}^{2}}{2\left(d L_{T} / d R_{m}\right)^{2}}\left[\frac{d^{2} E_{T, \mathrm{sym}}}{d R_{m}^{2}}-\frac{d E_{T, \mathrm{sym}}}{d R_{m}} \frac{d^{2} V / d R_{m}^{2}}{d V / d R_{m}}\right], \\
& k_{A}=\frac{R_{m, A}^{2}}{2\left(d L_{T} / d R_{m}\right)^{2}}\left[\frac{d^{2} E_{T, \mathrm{sym}}}{d R_{m}^{2}}-\frac{d E_{T, \mathrm{sym}}}{d R_{m}} \frac{d^{2} A / d R_{m}^{2}}{d A / d R_{m}}\right] .
\end{aligned}
$$

Replacing the expressions of $E_{T}, L_{T}, V$, and $A$ previously obtained by us (see Supplemental Material of Ref. [21]), we can determine analytically approximated solutions of the stability coefficients along the constriction pathway in terms of the spontaneous curvature of the membrane $C_{0}$, the surface tension $\Sigma$, and the osmotic pressure difference $\Delta p$. In previous works $[19,20]$, we addressed this question in vesicles with zero spontaneous curvature, $C_{0}=0$, negligible membrane tension, $\Sigma=0$, and isotonic conditions (no pressure difference between internal and external media), $\Delta p=0$. In this particular case, the values of $k_{V, A}$ are negative during 
all processes, indicating the unstable character of symmetric constriction. Here, we generalize this previous work to more realistic situations by taking into account the additional effects of $C_{0}, \Sigma$, and $\Delta p$. This allow us to study whether it is possible to get stable symmetric constriction for appropriate combinations of these parameters.

Maintaining constant volume, the stability coefficient $k_{V}$ in Eq. (A21) can be expressed as

$$
k_{V}=-\pi^{2} \kappa \frac{k_{V}^{N}}{k_{V}^{D}},
$$

with the numerator and denominator given by

$$
\begin{aligned}
k_{V}^{N}= & \frac{6^{1 / 4} 2}{9(\Gamma+29)^{2} \Lambda^{1 / 4}}\left[3807+417 \Gamma+12 \Gamma^{2}+1494 \Lambda+162 \Gamma \Lambda+4 \Gamma^{2} \Lambda-1125 \Lambda^{2}-25 \Gamma \Lambda^{2}-15 C_{0} R_{m}\right. \\
& \left.-10 C_{0} R_{m} \Lambda+25 C_{0} R_{m} \Lambda^{2}\right] s^{1 / 2}-\frac{\pi}{288 \Lambda}\left[30 \Lambda^{2}+216 \Gamma \Lambda-144 \Lambda-1446^{1 / 2} \Gamma \Lambda^{1 / 2}\right] s+\cdots \\
k_{V}^{D}= & \frac{6^{3 / 4} \pi^{3}}{16 \Lambda^{3 / 4}} s^{1 / 2}-\frac{6^{1 / 2} \pi^{2}}{3(\Gamma+29)^{2} \Lambda^{1 / 2}}\left[33018+459 \Gamma+13 \Gamma^{2}-1365 \Lambda-25 \Gamma \Lambda-40 C_{0} R_{m}+40 C_{0} R_{m} \Lambda\right] s+\cdots
\end{aligned}
$$

Alternatively, if the constraint of constant area is considered, the corresponding stability coefficient $k_{A}$ in Eq. (A22) takes the form

$$
k_{A}=-\pi^{2} \kappa \frac{k_{A}^{N}}{k_{A}^{D}},
$$

with the numerator and denominator given by

$$
\begin{aligned}
k_{A}^{N}= & \frac{6^{1 / 4} 4}{9(\Gamma+29)^{3} \Lambda^{1 / 4}}\left[110403+17466 \Gamma+819 \Gamma^{2}+12 \Gamma^{3}+35266 \Lambda+5892 \Gamma \Lambda+278 \Gamma^{2} \Lambda+4 \Gamma^{3} \Lambda-25785 \Lambda^{2}\right. \\
& -1570 \Gamma \Lambda^{2}-25 \Gamma^{2} \Lambda^{2}+1220 \Lambda^{3}+20 \Gamma \Lambda^{3}-435 C_{0} R_{m}-15 C_{0} R_{m} \Gamma-330 C_{0} R_{m} \Lambda-10 C_{0} R_{m} \Gamma \Lambda+805 C_{0} R_{m} \Lambda^{2} \\
& \left.+25 C_{0} R_{m} \Gamma \Lambda^{2}-40 C_{0} R_{m} \Lambda^{3}\right] s^{1 / 2}-\frac{\pi}{288 \Lambda}\left[1446^{1 / 2} \Lambda^{1 / 2}(\Lambda-\Gamma)+36 \Gamma \Lambda-42 \Lambda^{2}-144 \Lambda\right] s+\cdots, \\
& k_{A}^{D}=\frac{6^{3 / 4} \pi^{3}}{8 \Lambda^{3 / 4}} s^{1 / 2}-\frac{6^{1 / 2} 2 \pi^{2}}{3(\Gamma+29)^{3} \Lambda^{1 / 2}}\left[85507+16254 \Gamma+836 \Gamma^{2}+13 \Gamma^{3}-37875 \Lambda-2020 \Gamma \Lambda-25 \Gamma^{2} \Lambda\right. \\
& \left.\quad+305 \Lambda^{2}+5 \Gamma \Lambda^{2}-1170 C_{0} R_{m}+40 C_{0} R_{m} \Gamma(\Lambda-1)+1180 C_{0} R_{m} \Lambda-10 C_{0} R_{m} \Lambda^{2}\right] s+\cdots
\end{aligned}
$$

These coefficients are written in terms of $\Lambda$ [Eq. (3)] and $\Gamma=\left(2-C_{0} R_{m}\right)^{2}+2 \Sigma R_{m}^{2} / \kappa-1$, as are the expressions of $E_{T}, L_{T}, V$, and $A$ involved [5].

As stated above, in the particular case of vesicles with zero spontaneous curvature, $C_{0}=0$, negligible membrane tension, $\Sigma=0$, and isotonic conditions, $\Delta p=0$, the coefficients $k_{V, A}$ are negative during the entire process (black line in Fig. 9): they converge down to the asymptotic limit $\lim _{s \rightarrow 0} k_{V, A}=-2.77 \kappa \approx 28 k_{B} T$ for a flexible membrane $\left(\kappa \approx 10 k_{B} T\right.$ ), and become progressively less unstable with increasing constriction approaching zero in the largeconstriction limit $\lim _{s \rightarrow 1} k_{V, A}=0$. We (Refs. [19,20]) previously studied this case. In the general case studied here, when $C_{0}, \Sigma$, and $\Delta p$ are not necessarily zero, we also see that symmetric constriction becomes progressively more stable with increasing constriction. Additionally, we obtain a new and interesting result: the symmetric constriction can be stable $\left(k_{V, A}>0\right)$ against lateral deformations for appropriate combinations of these parameters (see Figs. 9, 3, and 4).

The approximate analytical results of the stability coefficients reproduce the numerical solutions for low and intermediate constriction regimes, with better agreements for vesicles with positive surface tensions $(\Sigma>0)$, immersed in hypertonic media $(\Delta p>0)$ and with positive spontaneous curvatures $\left(C_{0}>0\right)$ (see Fig. 9). The analytical result for the case with $\Sigma R_{m}^{2} / \kappa=-0.3$ and with $C_{0}=\Delta p=0$ (cyan triangles in Fig. 9) differs from the exact numerical values more than the other cases. The reason is that this combination of parameters gives the closer-to-zero value of $\Lambda$ and the analytical formulas of the stability coefficients [Eqs. (A23)(A28)] diverge as $\Lambda$ approaches zero.

Deduction of the stability coefficients. Each lobe of the asymmetric shape is characterized by a different polar radius: one of them has a greater polar radius $R_{m}^{+}>R_{m}$ (the right lobe in Fig. 2) while the other has a smaller one $R_{m}^{-}<R_{m}$ (the left lobe in Fig. 2). We denote these changes as

$$
\Delta R_{m}^{ \pm}=R_{m}^{ \pm}-R_{m}
$$

Similarly, the total length $L_{T}$ in each lobe changes with respect to the symmetric case in the form

$$
\Delta L_{T}^{ \pm}=L_{T}\left(R_{m}^{ \pm}, R_{c}\right)-L_{T}\left(R_{m}, R_{c}\right) .
$$

These parameters are clearly indicated in Fig. 7. 

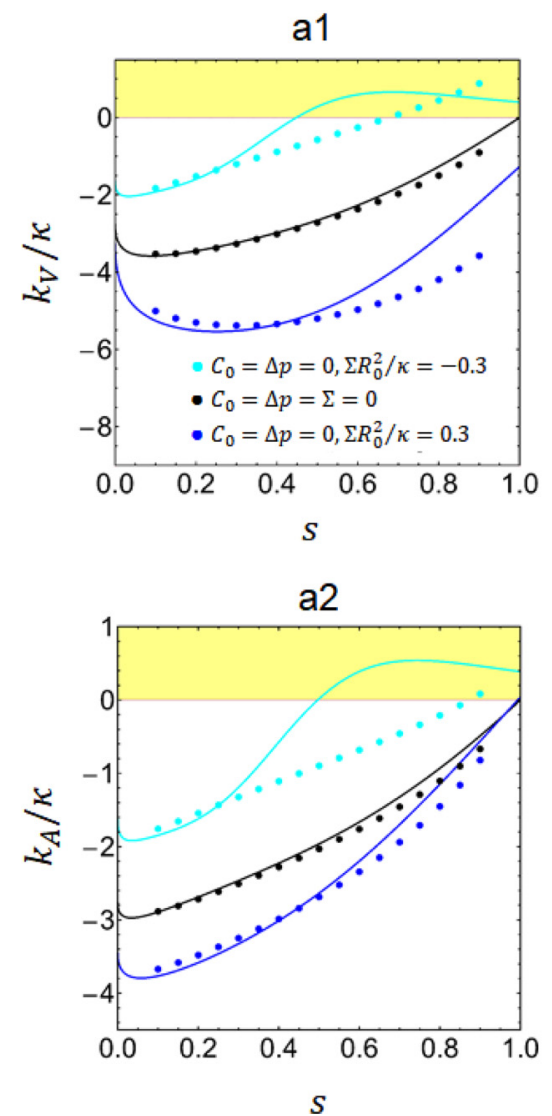

b1

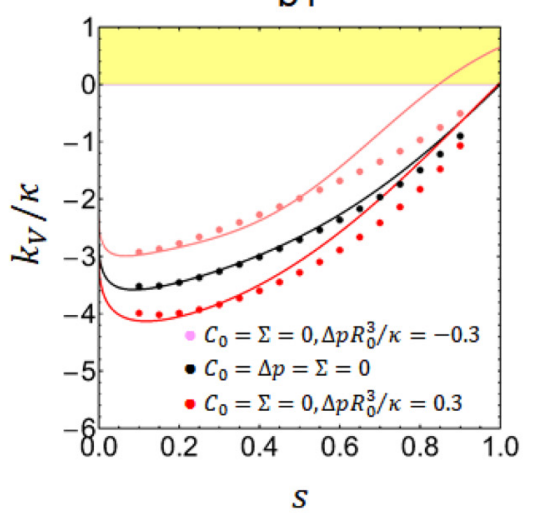

b2

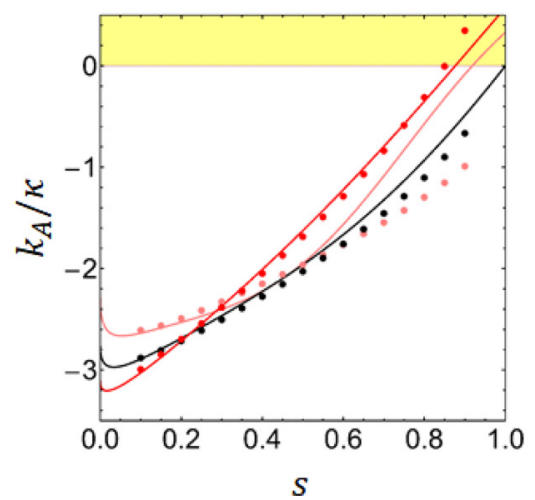

c1

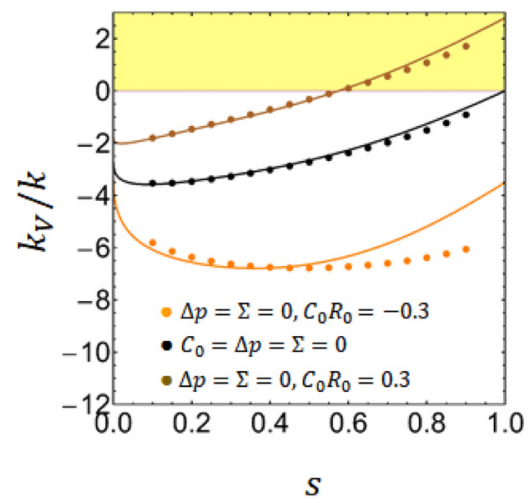

c2

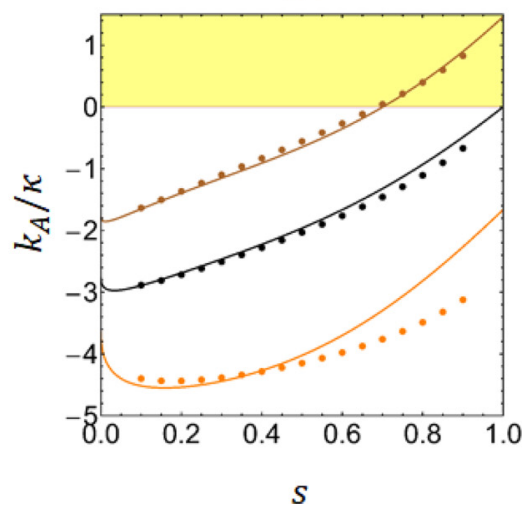

FIG. 9. Stability coefficients for constant volume and constant area constrictions. Stability coefficients maintaining volume constant $k_{V} / \kappa$ (above) and maintaining membrane area constant $k_{A} / \kappa$ (below) at all stages of constriction. Comparison of the exact numerical results with the analytical approximate solutions. (a) Influence of the surface tension $\Sigma$. (b) Influence of the osmotic pressure $\Delta p$. (c) Influence of the spontaneous curvature of the membrane $C_{0}$. Regions with stable configurations $\left(k_{V, A}>0\right)$ are shaded in yellow.

Stability coefficient at constant volume. If the transition between the symmetric and the asymmetric shapes occurs by keeping the volume constant, we can write

$$
V\left(R_{m}, R_{c}\right)=\frac{1}{2}\left[V\left(R_{m}+\Delta R_{m}^{-}, R_{c}\right)+V\left(R_{m}+\Delta R_{m}^{+}, R_{c}\right)\right] .
$$

This equation can be solved numerically which allows us to obtain the relation between $\Delta R_{m}^{+}$and $\Delta R_{m}^{-}$that maintains constant the volume enclosed by the vesicle. However, for small departures from the symmetrical shape $\Delta x$, an analytic perturbative computation is also valid. Expanding Eq. (A31) up to second order, we get

$$
V\left(R_{m}+\Delta R_{m}^{ \pm}, R_{c}\right)=V\left(R_{m}, R_{c}\right)+\frac{d V\left(R_{m}, R_{c}\right)}{d R_{m}} \Delta R_{m}^{ \pm}+\frac{1}{2} \frac{d^{2} V\left(R_{m}, R_{c}\right)}{d R_{m}^{2}}\left(\Delta R_{m}^{ \pm}\right)^{2}
$$

Introducing Eq. (A32) into Eq. (A31) gives

$$
0=\frac{1}{2}\left(\Delta R_{m}^{+}+\Delta R_{m}^{-}\right) \frac{d V\left(R_{m}, R_{c}\right)}{d R_{m}}+\frac{1}{4}\left[\left(\Delta R_{m}^{+}\right)^{2}+\left(\Delta R_{m}^{-}\right)^{2}\right] \frac{d^{2} V\left(R_{m}, R_{c}\right)}{d R_{m}^{2}} .
$$

Solving this quadratic equation for $\Delta R_{m}^{-}$and expanding the positive solution up to second order we have

$$
\Delta R_{m}^{-}=-\Delta R_{m}^{+}-\frac{d^{2} V / d R_{m}^{2}}{d V / d R_{m}}\left(\Delta R_{m}^{+}\right)^{2}
$$

The displacement of the constriction ring from the middle point of the symmetric shape $\Delta x$ can be related with the total lengths $L_{T}^{ \pm}$of the asymmetric poles as

$$
\Delta x\left(R_{m}, R_{c}\right)=\frac{1}{2}\left[L_{T}\left(R_{m}+\Delta R_{m}^{+}, R_{c}\right)-L_{T}\left(R_{m}+\Delta R_{m}^{-}, R_{c}\right)\right] .
$$


Expanding these lengths in Taylor series up to second order for small asymmetries we obtain

$$
L_{T}\left(R_{m}+\Delta R_{m}^{ \pm}, R_{c}\right)=L_{T}\left(R_{m}, R_{c}\right)+\frac{d L_{T}\left(R_{m}, R_{c}\right)}{d R_{m}} \Delta R_{m}^{ \pm}+\frac{1}{2} \frac{d^{2} L_{T}\left(R_{m}, R_{c}\right)}{d R_{m}^{2}}\left(\Delta R_{m}^{ \pm}\right)^{2} .
$$

Introducing Eqs. (A36) and (A34) into Eq. (A35) gives

$$
\Delta x\left(R_{m}, R_{c}\right)=\Delta R_{m}^{+} \frac{d L_{T}\left(R_{m}, R_{c}\right)}{d R_{m}}+\frac{\left(\Delta R_{m}^{+}\right)^{2}}{2} \frac{d^{2} L_{T}\left(R_{m}, R_{c}\right)}{d R_{m}^{2}} \frac{d^{2} V / d R_{m}^{2}}{d V / d R_{m}} .
$$

Solving for $\Delta R_{m}^{+}$,

$$
\Delta R_{m}^{+}=\frac{\Delta x}{d L_{T} / d R_{m}}-\frac{(\Delta x)^{2}}{2\left(d L_{T} / d R_{m}\right)^{2}} \frac{d^{2} V / d R_{m}^{2}}{d V / d R_{m}}
$$

Following the same steps, we can express the energy of the asymmetric shape in terms of the energies of the asymmetric lobes as

$$
E_{T, \text { asym }}\left(R_{m}, R_{c}\right)=\frac{1}{2}\left[E_{T, \text { sym }}\left(R_{m}+\Delta R_{m}^{-}, R_{c}\right)+E_{T, \text { sym }}\left(R_{m}+\Delta R_{m}^{+}, R_{c}\right)\right]
$$

Expanding it in power series up to second order for small asymmetries we have

$$
E_{T, \mathrm{sym}}\left(R_{m}+\Delta R_{m}^{ \pm}, R_{c}\right)=E_{T, \mathrm{sym}}\left(R_{m}, R_{c}\right)+\frac{d E_{T, \mathrm{sym}}\left(R_{m}, R_{c}\right)}{d R_{m}} \Delta R_{m}^{ \pm}+\frac{1}{2} \frac{d^{2} E_{T, \mathrm{sym}}\left(R_{m}, R_{c}\right)}{d R_{m}^{2}}\left(\Delta R_{m}^{ \pm}\right)^{2}
$$

Introducing the results of $\Delta R_{m}^{-}$[Eq. (A34)] and of $\Delta R_{m}^{+}$[Eq. (A38)] into Eq. (A40) we obtain that the difference of energy with respect to the symmetric configuration is given by a quadratic form as

$$
\Delta E_{T}=E_{T, \text { asym }}\left(R_{m}, R_{c}\right)-E_{T, \text { sym }}\left(R_{m}, R_{c}\right)=k_{V}\left(\frac{\Delta x}{R_{m, V}}\right)^{2},
$$

with the effective harmonic constant

$$
k_{V}=\frac{R_{m, V}^{2}}{2\left(d L_{T} / d R_{m}\right)^{2}}\left[\frac{d^{2} E_{T, \text { sym }}}{d R_{m}^{2}}-\frac{d E_{T, \text { sym }}}{d R_{m}} \frac{d^{2} V / d R_{m}^{2}}{d V / d R_{m}}\right] .
$$

Stability coefficient at constant area. Similarly, if in the transition between the symmetric and the asymmetric shapes the area is kept constant, the difference in their energies is given by the quadratic form

$$
\Delta E_{T}=E_{T, \text { asym }}\left(R_{m}, R_{c}\right)-E_{T, \text { sym }}\left(R_{m}, R_{c}\right)=k_{A}\left(\frac{\Delta x}{R_{m, A}}\right)^{2}
$$

with the effective harmonic constant

$$
k_{A}=\frac{R_{m, A}^{2}}{2\left(d L_{T} / d R_{m}\right)^{2}}\left[\frac{d^{2} E_{T, \mathrm{sym}}}{d R_{m}^{2}}-\frac{d E_{T, \mathrm{sym}}}{d R_{m}} \frac{d^{2} A / d R_{m}^{2}}{d A / d R_{m}}\right] .
$$

Useful relations between derivatives to numerically compute the stability coefficients. To obtain the stability coefficients of Eqs. (A42) and (A44) we have to calculate the following first and second derivatives with respect to $R_{m}$ :

$$
\frac{d L_{T}}{d R_{m}}, \frac{d E_{T}}{d R_{m}} \frac{d A}{d R_{m}}, \frac{d V}{d R_{m}}, \frac{d^{2} E_{T}}{d R_{m}^{2}} \frac{d^{2} A}{d R_{m}^{2}}, \frac{d^{2} V}{d R_{m}^{2}} .
$$

The numerical algorithm computes $E_{T}$ and the scaled quantities $L_{m} / R_{m}, A / R_{m}^{2}$, and $V / R_{m}^{3}$, which allows us to determine their first and second partial derivatives with respect to $s, C_{0} R_{m}, \Sigma R_{m}^{2}$, and $\Delta p R_{m}^{3}$, using the mean value theorem. To compute the total derivatives with respect to $R_{m}$, we can expand them into their partial derivatives as

$$
\begin{gathered}
\frac{d}{d R_{m}}=\frac{\partial s}{\partial R_{m}} \frac{\partial}{\partial s}+\frac{\partial\left(C_{0} R_{m}\right)}{\partial R_{m}} \frac{\partial}{\partial\left(C_{0} R_{m}\right)}+\frac{\partial\left(\Sigma R_{m}^{2}\right)}{\partial R_{m}} \frac{\partial}{\partial\left(\Sigma R_{m}^{2}\right)}+\frac{\partial\left(\Delta p R_{m}^{3}\right)}{\partial R_{m}} \frac{\partial}{\partial\left(\Delta p R_{m}^{3}\right)}=\frac{(1-s)}{R_{m}} \frac{\partial}{\partial s}+\Delta \\
\frac{d^{2}}{d R_{m}^{2}}=\frac{(1-s)^{2}}{R_{m}^{2}} \frac{\partial^{2}}{\partial s^{2}}-\frac{2(1-s)}{R_{m}^{2}} \frac{\partial}{\partial s}+\Delta^{2}
\end{gathered}
$$


using that $s=1-R_{c} / R_{m}$ and with

$$
\Delta=C_{0} \frac{\partial}{\partial\left(C_{0} R_{m}\right)}+2 \Sigma R_{m} \frac{\partial}{\partial\left(\Sigma R_{m}^{2}\right)}+3 \Delta p R_{m}^{2} \frac{\partial}{\partial\left(\Delta p R_{m}^{3}\right)}
$$

and

$$
\begin{aligned}
\Delta^{2}= & C_{0}^{2} \frac{\partial^{2}}{\partial\left(C_{0} R_{m}\right)^{2}}+4 \Sigma^{2} R_{m}^{2} \frac{\partial^{2}}{\partial\left(\Sigma R_{m}^{2}\right)^{2}}+2 \Sigma \frac{\partial}{\partial\left(\Sigma R_{m}^{2}\right)}+9 \Delta p^{2} R_{m}^{4} \frac{\partial^{2}}{\partial\left(\Delta p R_{m}^{3}\right)^{2}}+6 \Delta p R_{m} \frac{\partial}{\partial\left(\Delta p R_{m}^{3}\right)} \\
& +4 C_{0} \Sigma R_{m} \frac{\partial^{2}}{\partial\left(C_{0} R_{m}\right) \partial\left(\Sigma R_{m}^{2}\right)}+6 C_{0} \Delta p R_{m}^{2} \frac{\partial^{2}}{\partial\left(C_{0} R_{m}\right) \partial\left(\Delta p R_{m}^{3}\right)}+12 \Sigma \Delta p R_{m}^{3} \frac{\partial^{2}}{\partial\left(\Sigma R_{m}^{2}\right) \partial\left(\Delta p R_{m}^{3}\right)}+2 C_{0} \frac{(1-s)}{R_{m}} \frac{\partial^{2}}{\partial(s) \partial\left(C_{0} R_{m}\right)} \\
& +4 \Sigma(1-s) \frac{\partial^{2}}{\partial(s) \partial\left(\Sigma R_{m}^{2}\right)}+6 \Delta p(1-s) R_{m} \frac{\partial^{2}}{\partial(s) \partial\left(\Delta p R_{m}^{3}\right)}
\end{aligned}
$$

Now, we have to relate the partial derivatives of the scaled quantities computed numerically, $L_{T} / R_{m}, A / R_{m}^{2}$, and $V / R_{m}^{3}$, with the partial derivatives of the absolute quantities $L_{T}, A$, and $V$. After simple mathematical calculations, the partial derivatives with respect to $s$ are related as

$$
\begin{gathered}
\frac{\partial L_{T}}{\partial s}=R_{m} \frac{\partial\left(L_{T} / R_{m}\right)}{\partial s}+\frac{L_{T}}{(1-s)}, \\
\frac{\partial A}{\partial s}=R_{m}^{2} \frac{\partial\left(A / R_{m}^{2}\right)}{\partial s}+\frac{2 A}{(1-s)}, \\
\frac{\partial^{2} A}{\partial s^{2}}=R_{m}^{2} \frac{\partial^{2}\left(A / R_{m}^{2}\right)}{\partial s^{2}}+\frac{4}{(1-s)} \frac{\partial A}{\partial s}-\frac{2 A}{(1-s)^{2}}, \\
\frac{\partial V}{\partial s}=R_{m}^{3} \frac{\partial\left(V / R_{m}^{3}\right)}{\partial s}+\frac{3 V}{(1-s)}, \\
\frac{\partial^{2} V}{\partial s^{2}}=R_{m}^{3} \frac{\partial^{2}\left(V / R_{m}^{3}\right)}{\partial s^{2}}+\frac{6}{(1-s)} \frac{\partial V}{\partial s}-\frac{6 V}{(1-s)^{2}} .
\end{gathered}
$$

Note that the second derivatives of $L_{T}$ are not required since they are not involved in Eqs. (A42) and (A44). Finally, introducing the results of Eqs. (A50)-(A54) into Eqs. (A46) and (A47), we obtain the expressions of the total derivatives required to numerically determine the stability coefficients.

[1] B. M. Carlson, Principles of Regenerative Biology (Elsevier, San Diego, USA, 2007).

[2] D. O. Morgan, The Cell Cycle: Principles of Control (New Science Press, London, UK, 2007).

[3] K. Nasmyth, Viewpoint: Putting the cell cycle in order, Science 274, 1643 (1996).

[4] F. A. Barr and U. Gruneberg, Cytokinesis: Placing and making the final cut, Cell 131, 847 (2007).

[5] R. Rappaport, Establishment of the mechanism of cytokinesis in animal cells, Int. Rev. Cytol. 105, 245 (1986).

[6] P. Nurse, Eukaryotic cell-cycle control, Biochem. Soc. Trans. 20, 239 (1992).

[7] R. A. Daniel and J. Errington, Control of cell morphogenesis in bacteria: Two distinct ways to make a rod-shaped cell, Cell 113, 767 (2003).

[8] F. van den Ent, L. A. Amos, and J. Löwe, Prokaryotic origin of the actin cytoskeleton, Nature (London) 413, 39 (2001).

[9] A. L. Miller, The contractile ring, Curr. Biol. 21, R976 (2011).

[10] J. M. Ghuysen and R. Hakenbeck, Bacterial Cell Wall (Elsevier Science, Amsterdam, NL, 1994).
[11] J. A. Ayala, T. Garrido, M. A. de Pedro, and M. Vicente, Molecular biology of bacterial septation, New Compr. Biochem. 27, 73 (1994).

[12] M. A. de Pedro, W. D. Donachie, J.-V. Holtje, and H. Schwarz, Constitutive septal murein synthesis in Escherichia coli with impaired activity of the morphogenetic proteins RodA and penicillin-binding protein 2, J. Bacteriol. 183, 4115 (2001).

[13] D. W. Adams and J. Errington, Bacterial cell division: Assembly, maintenance and disassembly of the $\mathrm{Z}$ ring, Nat. Rev. Microbiol. 7, 642 (2009).

[14] H. P. Erickson, Modeling the physics of FtsZ assembly and force generation, Proc. Natl. Acad. Sci. USA 106, 9238 (2009).

[15] J. Xiao and E. D. Goley, Redefining the roles of the FtsZ-ring in bacterial cytokinesis, Curr. Opin. Microbiol. 34, 90 (2016).

[16] A. W. Bisson-Filho et al., Treadmilling by FtsZ filaments drives peptidoglycan synthesis and bacterial cell division, Science 355, 739 (2017).

[17] J. Sedzinski et al., Polar actomyosin contractility destabilizes the position of the cytokinetic furrow, Nature (London) 476, 462 (2011). 
[18] G. Salbreux, G. Charras, and E. Paluch, Actin cortex mechanics and cellular morphogenesis, Trends Cell Biol. 22, 536 (2012).

[19] V. G. Almendro-Vedia, F. Monroy, and F. J. Cao, Mechanics of constriction during cell division: A variational approach, PLoS One 8, e69750 (2013).

[20] V. G. Almendro-Vedia, F. Monroy, and F. J Cao, Analytical results for cell constriction dominated by bending energy, Phys. Rev. E 91, 12713 (2015).

[21] E. Beltrán-Heredia, V. G. Almendro-Vedia, F. Monroy, and F. $\mathrm{J}$ Cao, Modeling the mechanics of cell division: Influence of spontaneous membrane curvature, surface tension, and osmotic pressure, Front. Physiol. 8, 312 (2017).

[22] E. Fischer-Friedrich et al., Rheology of the active cell cortex in mitosis, Biophys. J. 111, 589 (2016).

[23] T. Kiyomitsu and I. M. Cheeseman, Cortical dynein and asymmetric membrane elongation coordinately position the spindle in anaphase, Cell 154, 391 (2013).

[24] P. B. Canham, The minimum energy of bending as a possible explanation of the biconcave shape of the human red blood cell, J. Theor. Biol. 26, 61 (1970).

[25] W. Helfrich, Elastic properties of lipid bilayers: Theory and possible experiments, Z. Naturforsch., C: J. Biosci. 28, 693 (1973).

[26] D. A. Fletcher and R. D. Mullins, Cell mechanics and the cytoskeleton, Nature (London) 463, 485 (2010).

[27] D. J. Steigmann, The Role of Mechanics in the Study of Lipid Bilayers (Springer International Publishing, New York, US, 2018).

[28] D. H. Boal, Mechanics of the Cell (Cambridge University Press, Cambridge, UK, 2002).

[29] H. T. McMahon and J. L. Gallop, Membrane curvature and mechanisms of dynamic cell membrane remodelling, Nature (London) 438, 590 (2005).

[30] U. Seifert, Configurations of fluid membranes and vesicles, Adv. Phys. 46, 13 (1997).

[31] D. J. Morre, Membrane biogenesis, Annu. Rev. Plant Physiol. 26, 441 (1975).

[32] A. Nohturfft and S. C. Zhang, Coordination of lipid metabolism in membrane biogenesis, Annu. Rev. Cell Dev. Biol. 25, 539 (2009).

[33] R. Albertson, B. Riggs, and W. Sullivan, Membrane traffic: A driving force in cytokinesis, Trends Cell Biol. 15, 92 (2005)

[34] E. Boucrot and T. Kirchhausen, Endosomal recycling controls plasma membrane area during mitosis, Proc. Natl. Acad. Sci. USA 104, 7939 (2007).

[35] Z. Kutalik, M. Razaz, A. Elfwing, A. Ballagi, and J. Baranyi, Stochastic modelling of individual cell growth using flow chamber microscopy images, Int. J. Food Microbiol. 105, 177 (2005).

[36] G. W. Niven, J. S. Morton, T. Fuks, and B. M. Mackey, Influence of environmental stress on distributions of times to first division in Escherichia coli populations, as determined by digital-image analysis of individual cells, Appl. Environ. Microbiol. 74, 3757 (2008).

[37] H.-J. Kim et al., Systemic analysis of heat shock response induced by heat shock and a proteasome inhibitor MG132, PLoS One 6, e20252 (2011)

[38] D. Raucher and M. P. Sheetz, Characteristics of a membrane reservoir buffering membrane tension, Biophys J. 77, 1992 (1999).

[39] M. P. Stewart et al., Hydrostatic pressure and the actomyosin cortex drive mitotic cell rounding, Nature (London) 469, 226 (2011).

[40] G. Murtas, Early self-reproduction, the emergence of division mechanisms in protocells, Mol. BioSyst. 9, 195 (2013).

[41] R. Rappaport, Cell division: Direct measurement of maximum tension exerted by furrow of echinoderm eggs, Science 156, 1241 (1967).

[42] A. Zumdieck, K. Kruse, H. Bringmann, A. A. Hyman, and F. Jülicher, Stress generation and filament turnover during actin ring constriction, PLoS One 2, e696 (2007).

[43] J. Käs and E. Sackmann, Shape transitions and shape stability of giant phospholipid vesicles in pure water induced by area-tovolume changes, Biophys. J. 60, 825 (1991).

[44] T. Baumgart, S. T. Hess, and W. W. Webb, Imaging coexisting fluid domains in biomembrane models coupling curvature and line tension, Nature (London) 425, 821 (2003).

[45] U. Seifert, K. Berndl, and R. Lipowsky, Shape transformations of vesicles: Phase diagram for spontaneous- curvature and bilayer-coupling models, Phys. Rev. A 44, 1182 (1991).

[46] P. Sens and M. S. Turner, Budded membrane microdomains as tension regulators, Phys. Rev. E 73, 031918 (2006).

[47] T. Kohyama, D. M. Kroll, and G. Gompper, Budding of crystalline domains in fluid membranes, Phys. Rev. E 68, 061905 (2003).

[48] R. A. Green, E. Paluch, and K. Oegema, Cytokinesis in animal cells, Annu. Rev. Cell Dev. Biol. 28, 29 (2012).

[49] M. K. Balasubramanian, E. Bi, and M. Glotzer, Comparative analysis of cytokinesis in budding yeast, fission yeast and animal cells, Curr. Biol. 14, R806 (2004).

[50] H. L. Sweeney and A. Houdusse, Structural and functional insights into the myosin motor mechanism, Annu. Rev. Biophys. 39, 539 (2010).

[51] O.-Y. Zhong-can and W. Helfrich, Instability and Deformation of a Spherical Vesicle by Pressure, Phys. Rev. Lett. 59, 2486 (1987).

[52] O.-Y. Zhong-can and W. Helfrich, Bending energy of vesicle membranes: General expressions for the first, second, and third variation of the shape energy and applications to spheres and cylinders, Phys. Rev. A 39, 5280 (1989).

[53] See Supplemental Material at http://link.aps.org/supplemental/ 10.1103/PhysRevE.100.052408 for MATHEMATICA code files generating the analytical and numerical results. 